Invited Review, Publications of the Astronomical Society of

the Pacific

\title{
The Distribution Of Heavy Elements In Spiral And Elliptical Galaxies
}

\author{
R.B.C. Henry \\ Department of Physics \& Astronomy, University of Oklahoma, Norman, OK 73019; \\ henry@mail.nhn.ou.edu \\ and \\ Guy Worthey \\ Department of Physics \& Astronomy, St. Ambrose University, Davenport, IA, 52803; \\ gworthey@saunix.sau.edu
}

\begin{abstract}
In large disk and spheroidal galaxies spatially resolved abundance information can be extracted by analysis of either emission lines, absorption lines, or both, depending on the situation. This review recaps significant results as they apply to non-dwarf galaxies, including the Milky Way, spiral disks and bulges, and elliptical and lenticular galaxies. Methods for determining abundances are explained in appendices.

Conclusions that span the galaxy types treated here are as follows. All galaxies, on average, have heavy element abundances (metallicities) that systematically decrease outward from their galactic centers while their global metallicities increase with galaxy mass. Abundance gradients are steepest in normal spirals and are seen to be progressively flatter going in order from barred spirals, lenticulars, and ellipticals. The distribution of abundances $N(Z)$ vs. Z is strongly peaked compared to simple closed-box model predictions of chemical enrichment in all galaxy types. That is, a "G dwarf problem", commonly known in the solar cylinder, exists for all large galaxies.

For spiral galaxies, local metallicity appears to be correlated with total (disk plus bulge) surface density. Examination of $\mathrm{N} / \mathrm{O}$ versus $\mathrm{O} / \mathrm{H}$ in spiral disks indicates that production of $\mathrm{N}$ is dominated by primary processes at low metallicity and secondary processes at high metallicity. Carbon production increases with increasing metallicity. Abundance ratios $\mathrm{Ne} / \mathrm{O}, \mathrm{S} / \mathrm{O}$, and $\mathrm{Ar} / \mathrm{O}$
\end{abstract}


appear to be universally constant and independent of metallicity, which argues either that the IMF is universally constant or that these ratios are not sensitive to IMF variations. In the Milky Way, there is a rough age-metallicity trend with much scatter, in the sense that older stars are more metal-poor.

In elliptical galaxies, nuclear abundances are in the range $[\mathrm{Z} / \mathrm{H}]=0.0$ to 0.4 , but the element mixture is not scaled-solar. In large elliptical galaxies $[\mathrm{Mg} / \mathrm{Fe}]$ is in the range 0.3 to 0.5 , decreasing to $\approx 0$ in smaller elliptical galaxies. Other light elements track the Mg enhancement, but the heavier Ca tracks Fe. Velocity dispersion appears to be a key parameter in the modulation of $[\mathrm{Mg} / \mathrm{Fe}]$, but the cause of the connection is unclear.

Subject headings: Galaxy: abundances — Galaxy: evolution — galaxies: abundances — galaxies: elliptical — galaxies: evolution — galaxies: ISM — galaxies: spiral — ISM: abundances

\section{Introduction}

Galactic chemical evolution is the proportional buildup of helium and heavy elements or metals, i.e. elements other than hydrogen and helium, within a galaxy over time as a result of the continuous manufacture and expulsion of these elements by resident stars. The topic concerns itself not only with global or pan-galactic changes but also with regional ones within spatially-resolved galaxies.

The essence of chemical evolution can be illustrated by imagining a closed box containing interstellar gas of primordial composition situated at an arbitrary location within a galaxy. As portions of the gas collapse, fusion processes within the stars that are formed convert hydrogen into heavier elements, and this chemically enriched material is subsequently expelled into the interstellar medium through stellar winds, planetary nebula formation, or supernova eruptions. As this cast-off matter mixes with the surrounding medium, the composition of the latter changes such that the abundances of helium and the heavy elements increase relative to hydrogen. As a second generation of stars forms from this enriched material, the new stars possess a greater fraction of heavy elements than their predecessors. Thus, the enrichment cycle continues until enough material has been locked up in stellar remnants that the star formation process, which depends upon the availability of interstellar gas, is finally damped. A galaxy, then, can be thought of as an ensemble of these boxes. 
The real picture is more complicated, of course. The boxes have no walls, and as such are open to matter exchange with their surroundings in all directions. Nevertheless, the simple model does suggest some of the crucial processes which must be understood if we are to have a comprehensive understanding. For example, we must know the details of star formation and evolution, stellar nucleosynthesis and the rate of heavy element production, the details of stellar death and matter ejection, and the efficiency with which ejected material is mixed into the interstellar medium.

Studies of galactic chemical evolution involve an interplay between 1) global and/or spatially resolved abundances, sometimes as a function of time, for one or more galaxies; and 2) models based upon a set of input parameters determined by the physics being tested. Observed abundances provide two basic sorts of information. First, ratios of heavy elements relative to hydrogen, such as $\mathrm{O} / \mathrm{H}$ or $\mathrm{Fe} / \mathrm{H}$, serve as gauges of how far chemical evolution has progressed in a system, because they measure the extent to which hydrogen has been converted to heavier elements. As such, these ratios are particularly sensitive to the rate at which gas is cycled through stars, i.e. the star formation rate, and how that rate may have changed with time. Second, ratios of two heavy elements, such as N/O or O/Fe, provide information about differential elemental production by stars. That is, at what rate, say, is nitrogen produced relative to oxygen, or oxygen relative to iron? The answer here is tied to the production rates of individual elements as a function of stellar mass weighted by the relative number of stars at each mass (the initial mass function; IMF) as well as to the history of star formation. Also, there is an element of time involved in all of this. For example, abundances measured in a star reveal enrichment levels at the time the star formed. In summary, chemical evolution can be traced indirectly by associating abundance patterns within a galaxy with local conditions, where the latter ultimately depend on time, and directly by observing abundances in stars of different ages or in galaxies of different look-back times.

The primary goal of this review is to describe the state of affairs concerning observed abundance patterns in galaxies. Because of author expertise, emphasis is placed on abundance patterns in spiral disks and elliptical galaxies as derived from emission line analyses and photometric indices, respectively. However, for completeness and continuity, we also describe and compare the complimentary results provided by stellar abundance work in the Milky Way and nearby galaxies. Our elemental scope is confined to those elements between carbon and iron on the periodic table $(6 \leq \mathrm{Z} \leq 26)$, i.e. those elements which are the most readily observed and for which there is the most information. Discussions of helium and the light elements are better taken up in the context of Big Bang nucleosynthesis, and for this the reader is urged to consult Chapter 4 of Pagel (1997) and references therein for recent discussions of this topic. Likewise, elements beyond iron have been studied in part 
by Edvardsson et al. (1993), Wheeler, Sneden, \& Truran (1989), and McWilliam (1997).

Numerous reviews of galactic chemical evolution and abundance patterns are available in the literature. An excellent, approachable introduction to the subject of chemical evolution is given in the comprehensive review by Tinsley (1980). The textbook by Pagel (1997) treats numerous topics related to galactic chemical evolution and the synthesis of elements. Additional material on observations and abundance studies in galaxies can be found in several recent conference proceedings, in particular Friedli et al. (1998) and Walsh \& Rosa (1999). Other useful works specifically treating element synthesis include books by Clayton (1983), Rolfs \& Rodney (1988) and Cowley (1995), the review by Trimble (1991), and the conference proceedings by Edmunds \& Terlevich (1992) and Prantzos, Vangioni-Flam, \& Cassé (1993). Finally, QSO absorption line systems are enabling chemical evolution studies to be carried out through the study of abundances as a function of look-back time. While these systems are beyond our scope, interested readers are urged to consult Lauroesch et al. (1996), Lu et al. (1996), and Pettini et al. (1999).

We begin with a discussion of abundances derived from emission lines in spiral galaxies, including the Milky Way, in $\S 2$. In $\S 3$ we turn to stars both inside and outside of the Milky way, while abundances in elliptical galaxies from photometric integrated light are treated in $\S 4$. A summary is given in $\S 5$. Appendices explain techniques used to derive abundances from emission lines (Appendix A), stellar absorption lines (Appendix B), and the integrated starlight of composite systems (Appendix C). Unless otherwise stated, elemental abundances and ratios referred to in this review are by number, not mass.

\section{Abundance Patterns In Spiral Galaxies From Emission-Line Objects}

Sampling a galaxy's interstellar medium directly provides a snapshot of the current abundance picture at the location being tested, in contrast to stellar abundances which for the most part are indicative of interstellar abundances at the time that the star formed. The most straightforward way of determining interstellar abundances is through the analysis of emission spectra produced by gas heated by nearby hot stars with continua rich in photons

having wavelengths shortward of $912 \AA$, i.e. the ionization edge of hydrogen. Such stars have effective temperatures exceeding $30,000 \mathrm{~K}$ and spectroscopically belong to the $\mathrm{O}$ and early $\mathrm{B}$ classes. Object types with these conditions include $\mathrm{H}$ II regions and planetary nebulae, reviews of which can be found in Shields (1990), Vila-Costas \& Edmunds (1992), and Zaritsky, Kennicutt, \& Huchra (1994) for H II regions and Peimbert (1990), Henry (1990), Perinotto (1991), Clegg (1993), and Habing \& Lamers (1997) for planetary nebulae. Old supernova remnants in which the stellar ejecta have completely mixed with the interstellar 
medium in principle represent a third type of emission line probe, since in this case the interstellar gas is heated by the shockwave producing emission lines. Often, however, full abundance studies are precluded by limited spectral coverage even within the optical (W.P. Blair, private communication), and thus there are far fewer abundance results available.

Ionized gases of the types just mentioned maintain temperature equilibrium in most cases by radiating photons at discrete wavelengths following recombination or collisional excitation processes involving ion-electron encounters. Spectra of these objects can then be analyzed to provide abundance, temperature, and density information. Measured from the ground in most cases, the strengths of the resulting emission lines can be converted to ionic and elemental abundances of $\mathrm{He}, \mathrm{C}, \mathrm{N}, \mathrm{O}, \mathrm{Ne}, \mathrm{S}$, and Ar using techniques described in Appendix A, which includes a table listing a number of prominent emission features. While the resulting abundances refer to levels in the gas phase only, Savage \& Sembach (1996) indicate that none of these elements is expected to be heavily partitioned into the solid phase in the form of dust. Thus, gas phase abundances should represent total values reasonably well.

Due to their size and therefore their accessibility in external galaxies, most of the abundance data from emission-line systems useful in chemical evolution studies relate to H II regions, which because of their association with recent star formation are located in spiral disks and irregular galaxies. This section focuses on abundance patterns in spirals.

It should be noted that abundances discussed are taken directly from the sources listed; no attempt has been made to homogenize them by recalculating the abundances in a consistent way. In general, differences in techniques and atomic data employed produce ranges in abundances which are smaller than observational uncertainties in the line strengths, and we believe that presenting unhomogenized data still provides a realistic representation of patterns and an opportunity to see the big picture.

\subsection{Metallicity Gradients In Spiral Disks}

Metallicity is the fraction by mass of all elements heavier than He in a system and is the primary indicator of chemical evolution as stars convert $\mathrm{H}$ into heavier elements and seed their environments with the products. Oxygen is the metallicity tracer of choice in the interstellar medium. Cosmically, its relative abundance surpasses all elements but $\mathrm{H}$ and He. Its relatively small depletion (Snow \& Witt 1996; Savage \& Sembach 1996) means it is present almost entirely in the gas phase. Hot, ionized gas in the vicinity of hot stars or

energetic shock waves give rise to H II regions, planetary nebulae, or supernova remnants 
whose spectra usually display prominent emission lines of oxygen. This contrasts sharply with the situation for old stars, for example, where absorption features of iron (heavily depleted onto grains in the interstellar medium) are prominent in stellar spectra due to the presence of optimal temperatures. Thus, iron is usually employed as a metallicity indicator when old stars are the probes.

Differences in the appearances of H II region spectra as a function of galactocentric distance were first noticed by Aller (1942) in his study of M33. Thirty years later, Searle (1971) connected similar differences across disks of several Sc galaxies with systematic changes in heavy element abundances. Early abundance gradient work in spirals is reviewed by Pagel \& Edmunds (1981), while the more contemporary picture is available in Friedli et al. (1998).

\subsubsection{Metallicity Gradients In The Milky Way Galaxy}

The disk of the Milky Way Galaxy (MWG) is arguably the most active and rapidly evolving region of our galaxy in the chemical sense. Probing it, though, is complicated by the presence of dust along all lines of sight within the disk, preventing radiation, particularly in the ultraviolet, from readily penetrating it. From our location within the disk, observing emission line objects is restricted not only from effects of reddening, but the presence of dust limits the distance over which we can probe. Despite these restrictions, large amounts of data are now available for H II regions and planetary nebulae within the disk of the MWG. The distance range from the sun in both the directions of the galactic center and anticenter has been extended by observations in the infrared, where extinction is at a minimum.

Table 1 summarizes the data for emission line objects in the disk of the MWG and compiled here. The columns in order indicate the type of object and spectral region (optical, far infrared, or radio) followed by the name of the first author on the paper for the data source, the total number of and galactocentric distance range for objects included in

each study, and finally an indication of the number of data points for each abundance ratio which were available in each study.

Six of the eight studies in Table 1 are based upon H II regions. The seminal study by Shaver et al. (1983) explored a range in galactocentric distance centered on the sun. More recent studies by Fich \& Silkey (1991), Vílchez \& Esteban (1996), and Rudolph et al. (1997) focused on the anti-center direction, while Simpson et al. (1995) and Afflerbach et al. (1997) studied objects toward the center of the Galaxy. Although the optical studies were 
all ground-based, the far infrared work in all three cases was carried out using the Cryogenic Grating Spectrometer aboard the Kuiper Airborne Observatory to observe emission lines such as [N III] $57 \mu \mathrm{m}$, [O III] $52,88 \mu \mathrm{m}$, and [S III] 19,33 $\mu \mathrm{m}$.

The remaining two papers in Table 1 were based on studies of planetary nebulae (Maciel \& Köppen 1994) and supernova remnants (Fesen, Blair, \& Kirshner 1985). Planetary nebulae comprise ejected material from evolved intermediate mass stars, and thus generally have an abundance profile which differs somewhat from that of the progenitor star at the time of birth due to contamination of the nebula by products of stellar nucleosynthesis. However, the contamination affects primarily helium, carbon, and nitrogen, and thus abundances of oxygen, sulfur, and argon in the nebula are expected to be good measures of the levels of those elements in the nascent progenitor star. In particular, Type II planetary nebulae (Peimbert 1978) have progenitors of small enough mass that oxygen is not expected to have been altered by CNO processing, yet they are disk objects, based upon their kinematics. Finally, the study of old supernova remnants by Fesen et al. measured abundances in disk objects over a radial range similar to that of Shaver et al.

Figure 1 plots $\mathrm{O} / \mathrm{H}$ versus galactocentric distance in kiloparcsecs by author, where $12+\log (\mathrm{O} / \mathrm{H})$ is used to represent oxygen 1 . Representative error bars are shown in the lower left panel. Solid lines show first order least squares fits for which the fitting parameters are given in Table 2, with the gradient $\mathrm{G}$ expressed in $\mathrm{dex} / \mathrm{kpc}$, the absolute abundance $\mathrm{A}_{8.5}$ given as $12+\log (\mathrm{O} / \mathrm{H})$ at the solar circle $(8.5 \mathrm{kpc})$, and $\mathrm{c}$ is the correlation coefficient. Dot-dashed lines show the composite fit from Table 2 for reference. Note that reported upper and lower limits on abundance ratios were generally not used in our compilation. We also point out that the larger scatter in the Simpson et al. data is probably due in large part to inferring $\mathrm{O} / \mathrm{H}$ from $\mathrm{O}^{+2} / \mathrm{S}^{+2}$ observations plus an assumption of a constant $\mathrm{S} / \mathrm{O}$ ratio, while the scatter in the Fesen et al. results for supernova remnants may be due to their using abundance-line strength diagnostic diagrams taken from shock models in the literature for estimating abundances.

Combining several data sets allows a visual comparison to be made among them as well as a test of the robustness of the trend exhibited by a single set. Figure 2 shows $12+\log (\mathrm{O} / \mathrm{H})$ versus galactocentric distance in $\mathrm{kpc}$ for the nebular data sets of Shaver et al., Afflerbach et al., Maciel \& Köppen, Vílchez \& Esteban, Fich \& Silkey, Rudolph et al., and Fesen et al. We also present the B star results from Smartt \& Rolleston (1997;

\footnotetext{
${ }^{1} \mathrm{H}$ II region distances in Shaver et al.'s data have been recomputed using their reported radial velocities and longitudes, eq. 9.3 in Binney \& Merrifield (1998), and assumptions that $\mathrm{R}_{\odot}=8.5 \mathrm{kpc}$ and the average circular velocity is $240 \mathrm{~km} / \mathrm{s}$ across the relevant portion of the disk.
} 
filled circles) and Gummersbach et al. (1998; open circles). The sun's position (Grevesse \& Noels 1993) is indicated with an ' $x$ ', while the error bars in the lower left show typical observational uncertainties for all of the data. A monotonic decrease in oxygen abundance with galactocentric distance is clearly present in the Milky Way disk. A simple least squares fit to all points except the B stars indicates a slope of $-0.06( \pm 0.01), A_{8.5}$ of 8.68 $( \pm 0.05)$, and a correlation coefficient of -0.63 . The data from Afflerbach et al. and Vilchez \& Esteban extend the trend of the main body of data toward the galactic center and anti-center, respectively. The uncertainty of \pm 0.2 dex in oxygen abundance is consistent with observational uncertainty, and thus there is no indication of real abundance scatter at a constant radial distance, in accord with findings of Kennicutt \& Garnett (1996) in their study of M101.

Several additional points are illustrated in Fig. 2. First, notice that the B star oxygen abundance trend is not noticeably different from the one defined by nebular data; in fact their gradients are very similar to the nebular results. This represents a major development, as previous attempts to infer the disk $\mathrm{O} / \mathrm{H}$ distribution from B stars (Gehren et al. 1985; Fitzsimmons et al. 1992; Kilian-Montenbruck et al. 1994; Kaufer et al. 1994) indicated the absence of a gradient. Smartt \& Rolleston speculate that sample size was the culprit in obscuring the gradient in most of the previous studies.

Next, the oxygen abundance distribution implied by planetary nebulae is indistinguishable from the one from H II regions. This would seem to confirm the value of PNe to trace disk metallicity and at the same time perhaps reduce the concern about diffusion (Wielen et al. 1996), i.e. that positions of PN progenitors shift radially during their lives, and thus PN abundances do not represent ISM conditions at their present galactocentric distances.

An interesting wrinkle in the MWG metallicity gradient picture is the possibility that the gradient flattens beyond $10 \mathrm{kpc}$. Results for the three anti-center studies are presented in a single panel in Fig. 1. Vílchez \& Esteban conclude that their data support the presence of a flattened oxygen gradient in the outer galaxy, although results from Fich \& Silkey and Rudolph et al. neither support nor counter this claim. Recently, Maciel \& Quireza (1999) have expanded and updated their sample to include PNe with larger galactocentric distances than those presented here. Like Vílchez \& Esteban above, they find evidence for a gradient which flattens beyond $12 \mathrm{kpc}$. A flattened gradient is both a controversial and interesting conjecture and is tied to the dynamics and mass distributions in the disk (Zaritsky 1992; Mollá et al. 1996; Samland, Hensler, \& Theis 1997), and we briefly return to this point in $\S 2.4$ in our general discussion of spiral abundance gradients.

Finally, we have plotted predictions of four chemical evolution models of the present-day 
disk for comparison with the data. The dashed line shows an analytical result based on the "simple model" from Pagel (1997; eq. 8.14), while detailed numerical model results are shown from Samland, Hensler, \& Theis (1997; solid line), Ferrini et al. (1994; dot-dashed line), and Köppen (1994; long-dashed line), where Köppen (private communication) employed a quadratic star formation law but recalculated his model for a radial flow velocity of $0.3 \mathrm{~km} / \mathrm{s}$. All models are scaled so as to match our composite interstellar oxygen abundance of 8.68 at the solar circle (see Table 2). Note that the Samland et al. model predicts a gradient flattening outward from around $11 \mathrm{kpc}$, the result of (according to them) mass loss of long-living metal-poor intermediate mass stars and additional infall of low metallicity gas in equilibrium with metal enrichment from condensation of intercloud medium.

\subsubsection{Metallicity Gradients In External Galaxies}

Results from numerous surveys of spiral galaxy abundance patterns show clearly that most spiral disks possess negative gradients qualitatively similar to the one in the Milky Way. Large surveys of $\mathrm{O} / \mathrm{H}$ in extragalactic H II regions include those of Mc Call (1982; 40 galaxies; see also Mc Call, Rybski, \& Shields 1985), Vila-Costas \& Edmunds (1992; 32 galaxies), and Zaritsky, Kennicutt, \& Huchra (1994; 39 galaxies). We can add to those the recent studies by Ferguson, Gallagher, \& Wyse (1998) and van Zee et al. (1998), both of which explored the outer regions of spirals, where star formation rates are much lower and the regions are less advanced chemically. Vila-Costas \& Edmunds reprocess reduced line strengths from the literature to obtain their abundances, while the other authors use primarily their own data for their studies. All these studies are based on optical spectra.

As an example of abundance patterns in two external spirals, in Fig. 3 we present a comparison of results for NGC 628 and M33 from Zaritsky et al. (1994) with Milky Way data from Shaver et al. (1983), Afflerbach et al. (1997), and Vílchez \& Esteban (1996), where $12+\log (\mathrm{O} / \mathrm{H})$ is plotted against galactocentric distance. The latter quantity has been normalized to the respective galaxy's isophotal radius $R_{\circ}$ [ to account for size variations

\footnotetext{
${ }^{2}$ The unpublished Köppen model also assumes a disk age of 15 Gyr along with exponentially decreasing infall both in time (5 Gyr scale) and galactocentric distance (4 kpc scale)

${ }^{3}$ The isophotal radius $\mathrm{R}_{\mathrm{O}}$ is the radial distance from the galactic nucleus at which the declining disk surface brightness reaches $25 \mathrm{mag} / \operatorname{arcsec}^{2}$. Comparisons of data among galaxies are made most frequently using the isophotal radius, but one could also use the effective radius, i.e. the radius of an aperture admitting one-half of the light from the disk, or kiloparsecs. To add to the confusion, literature sources for (non-nebular) bulge or elliptical galaxy abundances usually express gradients as $\Delta \log \mathrm{Z} / \Delta \log \mathrm{R}$ ! This last notation gives nonsensical
} 
among galaxies. $R_{\mathrm{o}}$ for the Milky Way disk was taken from de Vaucouleurs \& Pence (1978). Gradient slopes determined from least squares fits are given in the figure legend. The two external galaxies clearly resemble the Milky Way in possessing negative abundance grandients.

A much larger collection of abundance plots for individual spirals can be found in Zaritsky et al. (1994). We have extracted results from that paper and plotted them in Figure 4a, where characteristic abundancesf (top panels) and gradient slopes in $\operatorname{dex} / \mathrm{R}_{\mathrm{o}}$ (bottom panels) are shown as functions of galaxy morphological type ( $\mathrm{T}$ type), absolute blue magnitude $\mathrm{M}_{\mathrm{B}}$, and maximum circular velocity $\mathrm{V}_{\mathrm{c}}$ in $\mathrm{km} / \mathrm{s}$. All three of these parameters track galaxy mass, where smaller $\mathrm{T}$ type indices, more luminous integrated blue magnitudes, and larger rotational velocities generally correspond with more massive spirals. Normal (SA) and barred (SB) spirals are shown separately using filled and open symbols, respectively. Abundance parameters for the Milky Way composite fit from Table 2 are indicated in Fig. 4a with plusses, where we have adopted $\mathrm{T}=4, \mathrm{M}_{\mathrm{B}}=-20.08$, and $\mathrm{R}_{\mathrm{O}}=11.5 \mathrm{kpc}$ (de Vaucouleurs \& Pence 1978), along with $\mathrm{V}_{\mathrm{c}}=220 \mathrm{~km} / \mathrm{s}$ (Kochanek 1996).

Two important points are implied by Fig. 4a: (1) Characteristic abundances increase with galaxy mass, while gradient slopes are uncorrelated with this parameter; and

(2) Characteristic abundances in normal and barred spirals are indistinguishable, but barred spirals appear to have flatter (less negative) gradients. Both of these results have been noted previously. Garnett \& Shields (1987) plotted characteristic O/H values against galaxy mass for numerous spirals and found a direct correlation between these two parameters, while Pagel et al. (1979) first suggested that barred spirals may have flatter gradients, a pattern clearly borne out in the more extensive work by Martin \& Roy (1994), who relate gradient slope to bar strength, a quantity which measures bar ellipticity. Martin \& Roy find direct relations between the slope of the oxygen abundance gradient of a barred spiral and the galaxy's bar strength (ellipticity) and length in the sense that stronger bars are accompanied by flatter gradients. This empirical result is consistent with radial flow models of chemical evolution in which the presence of a bar enhances large-scale mixing over the galaxy's disk, damping radial abundance variations.

Interestingly, if gradient slope in dex/kpc (as opposed to dex $/ \mathrm{R}_{\mathrm{o}}$ shown here) is plotted versus $\mathrm{M}_{\mathrm{B}}$ (see Garnett 1998) a correlation appears such that more luminous galaxies have

abundances at the nucleus of a galaxy, but seems to match observed profiles out to the observational limit, which is usually far short of the isophotal radius for integrated starlight spectroscopy.

${ }^{4}$ The characteristic abundance is the abundance at $0.4 \mathrm{R}_{\mathrm{o}}$ as determined by a least squares fit to the data. See Zaritsky et al. (1994). 
flatter slopes. The dependence of slope behavior on normalization is no doubt related to the fact that $R_{o}$ for luminous galaxies tends to be longer in kiloparsecs. Since the vertical scatter in the lower panel of Fig. 4a is comparable to observational uncertainties, this may imply a universal gradient in $\operatorname{dex} / R_{\mathrm{o}}$, which in turn could be associated with similar timescales for viscous angular momentum transport and star formation, producing exponential gradients in surface density and abundances (Lin \& Pringle 1987; Yoshii \& Sommer-Larsen 1989).

We illustrate explicitly the correlation between galaxy mass and characteristic abundance in Fig. 4b, where we plot $12+\log (\mathrm{O} / \mathrm{H})$ at one effective radius versus the $\log$ of the galaxy mass in solar units for a sample of spiral galaxies. Abundance data are from Garnett \& Shields (1987), Skillman et al. (1996), and Henry et al. (1996). Sources for galaxy masses, which for the most part are inferred from rotation curves, are given in Henry et al. The two points connected by a horizontal line are for NGC 753 whose mass was determined for $\mathrm{H}_{\mathrm{o}}$ values of 50 and $100 \mathrm{~km} / \mathrm{s}$. The least squares fit to the data, shown with a solid line, indicates that $12+\log (\mathrm{O} / \mathrm{H})=3.79+0.47 \times \log \mathrm{M}$, where $\mathrm{M}$ is in solar masses. Note that this relation ignores the low surface brightness spirals (McGaugh 1994) which appear to have low metallicity but high mass. These objects are discussed briefly in $§ 2.1 .3$.

Finally, Figure 5 shows the observed relation between $12+\log (\mathrm{O} / \mathrm{H})$ and total (disk + bulge) surface density, $\Sigma$, in $\mathrm{M}_{\odot} / \mathrm{pc}^{2}$ at the corresponding location from Vila-Costas \& Edmunds (1992; their Fig. 7d), where open and closed squares represent H II regions residing in late (Scd-Irr) and early (Sab-Sc) spirals, respectively. Vila-Costas \& Edmunds assumed that the mass distribution follows the light distribution and employed a constant mass-to-light ratio for each galaxy, the latter determined from a rotation curve (see Vila-Costas \& Edmunds and Mc Call 1982 for details). The scatter is consistent with observational uncertainty, and thus we see a clear positive correlation between abundance and local surface density in spirals, with earlier spirals generally possessing higher abundances per unit surface density.

\subsubsection{Assorted Issues About Galaxy Metallicity}

Other issues concerning abundance gradients include questions about gradients perpendicular to the disk as well as azimuthal distributions, abundance patterns in low surface brightness galaxies, effects of cluster environment on gradients, the mathematical form of abundance profiles, and results of extragalactic planetary nebula studies. We treat these topics briefly. 
A negative vertical gradient in $O / H$ in the Milky Way is suggested by planetary nebula studies. Abundance data compiled by Kaler (1980) for PNe ranging in height above the disk from less than $0.4 \mathrm{kpc}$ to greater than $1 \mathrm{kpc}$ show a decrease in $\mathrm{O} / \mathrm{H}$ with increasing height above the plane. A comparison of more recent studies of PNe close to the plane (Perinotto 1991), greater than 300pc above the plane (Cuisinier et al. 1996), and in the halo (Howard, Henry, \& McCartney 1997) shows averages of $12+\log (\mathrm{O} / \mathrm{H})$ for these three samples of $8.68,8.52$, and 8.02 respectively, qualitatively consistent with Kaler.

Thorough tests for azimuthal gradients in spiral disks have yet to be carried out. One example of apparent $\mathrm{O} / \mathrm{H}$ asymmetry is discussed by Kennicutt \& Garnett (1996) in their study of M101. They find that H II regions located along a spiral arm southeast of the major axis have a lower oxygen abundance by 0.2-0.4 dex compared with H II regions on the opposite side.

Global metallicities in low surface brightness galaxies are generally found to be subsolar by roughly a factor of three, according to McGaugh (1994), indicating that these galaxies evolve very slowly and form few stars during a Hubble time. Apparently, they also lack detectable gradients. This, despite the fact that these objects are similar in mass and size to prominent spirals defining the Hubble sequence. McGaugh suggests that a galaxy's environment and surface mass density are more relevant to galaxy evolution than gross size.

Effects of cluster environment on the chemical evolution of galaxies have been investigated by Skillman et al. (1996), who studied oxygen profiles in several Virgo spirals representing a range in $\mathrm{H}$ I deficiency (taken as a gauge of cluster environmental interactions). Their results imply that global metal abundances in disks tend to be higher in stripped galaxies, presumably because reduced infall of metal-poor H I gas means less dilution of disk material. Henry et al. (1996 and references therein) investigated metallicity and heavy element abundance ratios $(\mathrm{N} / \mathrm{O}, \mathrm{S} / \mathrm{O})$ in three cluster spiral disks with normal $\mathrm{H}$ I and found no clear signatures of environmental effects. Thus, cluster environment alone is apparently not a sufficient condition for altered chemical evolution.

The mathematical form of abundance profiles in spiral disks has been investigated recently by Henry \& Howard (1995), who fit line strength behavior over the disks of M33, M81, and M101 using photoionization models. Their best fits for $\mathrm{O} / \mathrm{H}$ versus galactocentric distance were produced using exponential profiles, although power law forms could not be ruled out. However, linear profiles poorly reproduced the observations. Henry and Howard also concluded that, despite some observational and theoretical claims to the contrary (see Mollá et al. 1996), it is premature to conclude that gradient flattening is present in the outer parts of some disks. 
Planetary nebulae have been used as probes of interstellar abundances in a small number of external galaxies. A recent paper by Jacoby \& Ciardullo (1999) presents abundances for 12 bulge and three disk planetaries in M31. Their bulge objects have oxygen abundances whose average is similar to the value observed in the Large Magellanic Cloud. Interestingly, the implied bulge metallicity is significantly below the level expected from observations of $[\mathrm{Fe} / \mathrm{H}]$. In another study, Stasińska, Richer, \& Mc Call (1998) determine abundances of oxygen, neon, and nitrogen in planetaries in the bulges of the Milky Way and M31, M32, and the Magellanic Clouds. These authors find higher oxygen levels in the Milky Way and M31 bulges than in the Clouds, and also reconfirm the tight correlation between neon and oxygen discussed below in $\S 2.2 .3$.

\subsubsection{Summary Thoughts About Spiral Metallicities}

A detailed synthesis based upon the observations is beyond the scope of our review. However, the following would seem to provide a reasonable set of explanations.

There appear to be two fundamental physical parameters for a galaxy which influence its abundance characteristics. These are total mass and the distribution of material as a function of galactocentric distance. As supernovae erupt, their metal-rich ejecta are more likely retained in systems with greater mass. Thus, the more massive galaxies might be expected to exhibit higher global metallicities, which in fact they do. Furthermore, observations indicate that metallicities tend to be greater in regions where the total surface density is higher, perhaps because the star formation process is sensitive to the local density and so more metals are produced in locations with high densities. Since matter in spirals tends to form an exponential disk (Binney \& Merrifield 1998) with surface density falling off with greater galactocentric distance, we might then expect metallicity locally in the disk to track this pattern.

\subsection{Heavy Element Abundance Ratios In Spiral Disks}

Ratios of heavy elements, i.e. $\mathrm{N} / \mathrm{O}$ and $\mathrm{C} / \mathrm{O}$, are expected to reveal in particular the characteristics of the initial mass function, stellar yields, and the history of star formation. Here we consider five ratios which are accessible through nebular studies, N/O, C/O, Ne/O, $\mathrm{S} / \mathrm{O}$, and $\mathrm{Ar} / \mathrm{O}$. Note that because planetary nebulae are self-contaminating with nitrogen and (sometimes) carbon, they do not make good probes of the interstellar levels for these elements, although in the cases of $\mathrm{O}, \mathrm{Ne}, \mathrm{S}$, and Ar they seem to work satisfactorily in that 
capacity.

\subsection{1. $N / O$}

We consider the nitrogen abundance studies for the Milky Way disk indicated in Table 1 along with H II region studies by Kobulnicky \& Skillman (1996), van Zee et al. (1998), Thurston, Edmunds, \& Henry (1996), and Izotov \& Thuan (1999) for external spirals. Figure 6 shows $\log (\mathrm{N} / \mathrm{O})$ versus $12+\log (\mathrm{O} / \mathrm{H})$ for both the Milky Way and extragalactic objects. Symbols are explained in the caption.

The most striking feature in Fig. 6 is the apparent threshold running from the lower left to upper right beginning around $12+\log (\mathrm{O} / \mathrm{H})=8.25$ and breached by only a few objects. Behind this line the frequency of objects drops off toward lower values of $12+\log (\mathrm{O} / \mathrm{H})$ and higher values of $\log (\mathrm{N} / \mathrm{O})$. A second feature is the behavior of $\mathrm{N} / \mathrm{O}$ at values of $12+\log (\mathrm{O} / \mathrm{H})<8$, where $\mathrm{N} / \mathrm{O}$ appears constant, a trend which seems to be reinforced by the upper limits provided by the damped Ly $\alpha$ objects of Lu et al. (1996; L) at very low metallicity. This bi-modal behavior of N/O was pointed out by Kobulnicky \& Skillman (1996). Although detailed theoretical interpretations are beyond our scope, we summarize below the basic ideas of nitrogen production and attempt to tie them to Fig. 6. Readers interested in additional detail are urged to refer to Vila-Costas \& Edmunds (1993).

Nitrogen is mainly produced in the six steps of the $\mathrm{CN}$ branch of the CNO bi-cycle within $\mathrm{H}$ burning stellar zones, where ${ }^{12} \mathrm{C}$ serves as the reaction catalyst (see a textbook like Clayton 1983 or Cowley 1995 for nucleosynthesis review). Three reactions occur to transform ${ }^{12} \mathrm{C}$ to ${ }^{14} \mathrm{~N}:{ }^{12} \mathrm{C}(\mathrm{p}, \gamma){ }^{13} \mathrm{~N}\left(\beta^{+} \nu\right){ }^{13} \mathrm{C}(\mathrm{p}, \gamma){ }^{14} \mathrm{~N}$, while the next step, ${ }^{14} \mathrm{~N}(\mathrm{p}, \gamma) \mathrm{O}^{15}$, depletes nitrogen and has a relatively low cross-section. The final two reactions in the cycle transform ${ }^{15} \mathrm{O}$ to ${ }^{12} \mathrm{C}$. Since the fourth reaction runs much slower than the others, the cycle achieves equilibrium only when ${ }^{14} \mathrm{~N}$ accumulates to high levels, and so one effect of the $\mathrm{CN}$ cycle is to convert ${ }^{12} \mathrm{C}$ to ${ }^{14} \mathrm{~N}$. The real issue in nitrogen evolution is to discover the source of the carbon which catalyzes the process.

Since stars produce their own carbon during He burning, nitrogen originating from it is termed primary nitrogen. Any nitrogen produced during supernova explosive nucleosynthesis is also termed primary since it is created for the first time during the explosion. On the other hand, stars beyond the first generation in a galactic system already contain some carbon inherited from the interstellar medium out of which they formed. Nitrogen produced from this carbon is termed secondary nitrogen.

As a system begins to mature chemically from a state of low metallicity, nitrogen must 
come from carbon produced by the star itself, since at this point no significant level of carbon exists in the ISM which can be incorporated into new stars and enter into the CN cycle. So, nitrogen production is primary and its evolution proceeds at a rate set only by star formation coupled with the primary production rate of nitrogen. Since the production of elements such as oxygen is being influenced by similar factors, the N/O ratio should remain constant as their abundances rise together.

But as metallicity rises and stars form out of progressively more metal-rich environments, the amount of carbon present in the star at birth which can ultimately enter the CN cycle becomes comparable to the amount produced internally through He burning, and thus nitrogen production becomes secondary and coupled to the metallicity of the star. At this point, $\mathrm{N} / \mathrm{O}$ versus $\mathrm{O} / \mathrm{H}$ assumes a positive slope, since the relation between $\mathrm{N}$ and O is now quadratic (Vila-Costas \& Edmunds 1993).

Based upon the data and models presented in Fig. 6 and allowing for the scatter, a reasonable explanation for the observed trend for $\mathrm{N} / \mathrm{O}$ is that the flatter behavior seen at $12+\log (\mathrm{O} / \mathrm{H})<8.0$ corresponds to the dominance of primary nitrogen production, while the steeper slope in $\mathrm{N} / \mathrm{O}$ at higher metallcities is linked to metallicity-sensitive secondary nitrogen production. We concur with Shields, Skillman, \& Kennicutt (1991), who found that the point at which secondary nitrogen production becomes important is located at roughly $12+\log (\mathrm{O} / \mathrm{H})=8.3$ or 0.6 dex below solar.

A comparison of nitrogen yields from intermediate mass stars $\left(1-8 \mathrm{M}_{\odot}\right)$ by van den Hoek \& Groenewegen (1997) with those from massive stars by Nomoto et al. (1997b) suggests that intermediate mass stars are ultimately the main contributors to nitrogen production, although early-on massive stars may play a role, due to the longer evolutionary time scales for less massive stars, and thus their delay in depositing nitrogen into the interstellar medium. Vila-Costas and Edmunds (1993) calculated analytical models for the evolution of $\mathrm{N} / \mathrm{O}$ assuming a simple, closed-box regime but accounting separately for primary and secondary nitrogen yields along with time delays in intermediate-mass star nucleosynthesis. The two curves in Fig. 6 represent their results using their Eq. A5 along with two different values for the ratio of time delay to the system age. We have adopted values for constants a and b in their formula of 0.025 and 120 , respectively, to force a better fit to the data presented here. The curve representing the small delay clearly matches the low metallicity data better, while the curve for greater delay seems to rise faster at high metallicity and thus fit the data there better.

Further study of the origin of nitrogen will require especially more abundances for systems of low metallicity where $12+\log (\mathrm{O} / \mathrm{H})<7$. Studies of damped Lyman- $\alpha$ systems currently offer great promise in this regard. 


\subsection{2. $C / O$}

Carbon is produced during core and shell helium burning in the triple alpha process, $3^{4} \mathrm{He} \rightarrow{ }^{12} \mathrm{C}$. It is an element whose abundance has lately become more measurable in extra-galactic H II regions, thanks to the Hubble Space Telescope (HST) and its UV capabilities, since the strong carbon lines of C III] and C IV appear in that spectral region. Recent studies of extragalactic H II regions have been carried out by Garnett et al. (1995; 1997; 1999) and Kobulnicky \& Skillman (1998), while carbon abundances for M8 and the

Orion Nebula, both within the MWG, have been measured by Peimbert et al. (1993) and Esteban et al. (1998), respectively.

Results of these measurements are collected together in Fig. 7, where $\log (\mathrm{C} / \mathrm{O})$ is plotted against $12+\log (\mathrm{O} / \mathrm{H})$. The point for Orion is indicated with an ' $\mathrm{O}$ ', M8 with 'M', and the sun with an 'S' (Grevesse et al. 1996). The vertical lines connect points corresponding to carbon abundances determined with two different reddening laws by Garnett et al. (1998). The filled circles correspond to stellar data from Gustafsson et al. (1999) for a sample of $\mathrm{F}$ and $\mathrm{G}$ stars.

A direct correlation between $\mathrm{C} / \mathrm{O}$ and $\mathrm{O} / \mathrm{H}$ is strongly suggested and has been noted before (c.f. Garnett et al. 1999), although the result is weakened somewhat by the two points for $\mathrm{I} \mathrm{Zw} 18$ around $12+\log (\mathrm{O} / \mathrm{H})=7.25$. Ignoring these two points as well as the ones for the sun and stellar data, and performing a regression analysis, we find that $\log (\mathrm{C} / \mathrm{O})=-5.34( \pm 0.68)+0.59( \pm 0.08)[\log (\mathrm{O} / \mathrm{H})+12]$ (solid line in Fig. 7) with a correlation coefficient of 0.88 when we exclude Garnett et al.'s (1999) data points corresponding to $\mathrm{R}_{\mathrm{v}}=5$ (the connected points with lower $\mathrm{C} / \mathrm{O}$ ), $\log (\mathrm{C} / \mathrm{O})=-4.45( \pm 0.60)+0.48( \pm 0.07)[\log (\mathrm{O} / \mathrm{H})+12]($ dashed line in Fig. 7$)$ with a correlation coefficient of 0.86 when points for $R_{v}=3.1$ (the connected points with higher $\mathrm{C} / \mathrm{O}$ ) are excluded. Assuming that with additional data the trend becomes more robust, it clearly implies that carbon production is favored by higher metallicities. One promising explanation (Prantzos, Vangioni-Flam, \& Chauveau 1994; Gustafsson et al. 1999) is that mass loss in massive stars is enhanced by the presence of metals in their atmospheres which increase the UV cross-section to stellar radiation. Stellar yield calculations by Maeder (1992) appear to support this claim. The contributions to carbon by different stellar mass ranges is discussed by both Prantzos et al. and Gustafsson et al., who conclude that the massive stars are primarily responsible for carbon production. It is also clear, however, that stars of mass less than about $5 \mathrm{M}_{\odot}$ produce and expel carbon as well (van den Hoek \& Groenewegen 1997), and thus the relative significance of massive and intermediate mass stars is still not understood completely. 


\subsection{3. $\mathrm{Ne} / \mathrm{O}, \mathrm{S} / \mathrm{O}, \mathrm{E} \mathrm{Ar} / \mathrm{O}$}

Neon is produced through carbon burning $\left({ }^{12} \mathrm{C}+{ }^{12} \mathrm{C} \rightarrow{ }^{20} \mathrm{Ne}+{ }^{4} \mathrm{He}\right)$, while both sulfur and argon originate from explosive oxygen burning in Type II supernova events $\left({ }^{16} \mathrm{O}+{ }^{16} \mathrm{O} \rightarrow{ }^{28} \mathrm{Si}+{ }^{4} \mathrm{He}\right.$, then $\left.{ }^{28} \mathrm{Si}+{ }^{4} \mathrm{He} \rightarrow{ }^{32} \mathrm{~S} ;{ }^{32} \mathrm{~S}+{ }^{4} \mathrm{He} \rightarrow{ }^{36} \mathrm{Ar}\right)$. In addition, substantial amounts of $\mathrm{S}$ and Ar may be manufactured in Type Ia supernova events (Nomoto et al. 1997a). Note that here we refer only to the dominant isotopes of the respective elements.

Abundance ratios of $\mathrm{Ne} / \mathrm{O}, \mathrm{S} / \mathrm{O}$, and $\mathrm{Ar} / \mathrm{O}$ are plotted logarithmically against $12+\log (\mathrm{O} / \mathrm{H})$ in Fig. 8. To the data of Shaver et al. (1983) and Maciel \& Köppen (1994) for the Milky Way we have added data for $\mathrm{Ne} / \mathrm{O}, \mathrm{S} / \mathrm{O}$, and Ar/O from optical studies of extragalactic H II regions from van Zee et al. (1998) and Izotov \& Thuan (1999) along with S/O results from Garnett (1989) for both the MWG and extragalactic H II regions. Representative uncertainties are \pm 0.20 dex in each of the three ratios and \pm 0.20 dex in $12+\log (\mathrm{O} / \mathrm{H})$. The horizontal lines in each panel represent the predictions from Nomoto et al. (1997a; dashed lines), Woosley \& Weaver (1995; dot-dashed lines), and Samland (1998; solid lines) for massive star yields integrated over a Salpeter initial mass function between $10-50 \mathrm{M}_{\odot}$ and corrected to give ratios by number.

All three panels of Fig. 8 show vertical ranges which are consistent with uncertainties and thus imply in each case a constant value for each ratio over the 1.5-2 decades of oxygen abundance. Logarithmic values for unweighted arithmetic averages (log average antilog) and standard deviations (not uncertainties) for $\mathrm{Ne} / \mathrm{O}, \mathrm{S} / \mathrm{O}$, and $\mathrm{Ar} / \mathrm{O}$ are presented in Table 3, where the first column identifies the sample by the last name of the first author followed by three numbers indicating the sample sizes for $\mathrm{Ne} / \mathrm{O}, \mathrm{S} / \mathrm{O}$, and $\mathrm{Ar} / \mathrm{O}$, respectively. Also included are averages for the total of all samples, solar values (Grevesse et al. 1996), and ratios found in the Orion Nebula (Esteban et al. 1998) and the Helix Nebula (Henry, Kwitter, \& Dufour 1999), a nearby planetary nebula. Generally, for each abundance ratio there is very good agreement among the five samples, considering observational uncertainties. Notice the smaller dispersion associated with the Izotov \& Thuan data. This may be explained by their focus on metal-poor $\mathrm{H}$ II regions possessing very bright emission lines with resulting signal-to-noise of 20-40 in the continuum and abundances frequently having uncertainties of less than \pm 0.10 dex compared with the typical \pm 0.20 dex uncertainties in other samples (Izotov, private communication). In addition, we note the significant disparity between Ar/O for Orion and the other samples along with the sun and the Helix Nebula. Due to the limited spectroscopic range, the argon abundance in Orion was determined using the weak $5192 \AA$ auroral line of $\mathrm{Ar}^{+2}$, where its strength was observed to be on the order of $10^{-3}$ times $\mathrm{H} \beta$. Use of stronger near IR lines may bring the argon abundance in Orion into agreement with other objects (Esteban, private communication). 
In addition, the $\mathrm{S} / \mathrm{O}$ ratio found in the Helix Nebula is an order of magnitude below the average value. This is currently difficult to interpret, although a few planetary nebulae do show sulfur abundances which are this low (see Henry et al. 1999).

The evidence provided by Table 3 and Fig. 8 supports the contention that abundances of Ne, S, Ar, and O evolve in lockstep, a point made by Henry (1989) in his earlier study of $\mathrm{Ne}$ and $\mathrm{O}$ in planetary nebulae. This would be expected if these elements are all either produced by massive stars within a narrow mass range or stars of different masses but with an invariant initial mass function. Under these conditions their buildup is expected to proceed in lockstep, and the ratios of $\mathrm{Ne} / \mathrm{O}, \mathrm{S} / \mathrm{O}$, and $\mathrm{Ar} / \mathrm{O}$ should have constant values over a range of $\mathrm{O} / \mathrm{H}$.

Interesting departures from the universality of the ratios displayed in Fig. 8 appear in halo planetary nebula studies. The detailed one by Howard, Henry, \& McCartney (1997), for example, confirms earlier indications that the object BB-1 has $\log (\mathrm{Ne} / \mathrm{O})$ of -0.11 , while $\log (\mathrm{Ne} / \mathrm{O})$ for $\mathrm{H} 4-1$ has a value of -1.82 . These deviants might be explained by local abundance fluctuations caused by recent supernova events whose ejecta, differing in composition because of mass cut differences in the explosive event, had not yet mixed in with the surrounding ISM before the PN progenitor formed out of it.

Finally, notice that predicted ratios from the yields for stars in the $10-50 \mathrm{M}_{\odot}$ mass range, represented by the horizontal lines, generally fall below the observed average, with the offset for Nomoto et al. consistently being the largest. This suggests that the theoretical calculations overproduce oxygen and further imply that the adopted rate of the ${ }^{12} \mathrm{C}(\alpha, \gamma){ }^{16} \mathrm{O}$ reaction in the models is too high, resulting in a higher conversion rate of ${ }^{12} \mathrm{C}$ to ${ }^{16} \mathrm{O}$ with a boost in the oxygen production relative to elements such as Ne, S, and Ar. This conclusion agrees at least qualitatively with comparisons by Nomoto et al. (1997b) of yields from two $25 \mathrm{M}_{\odot}$ stellar models using significantly different values of the ${ }^{12} \mathrm{C}(\alpha, \gamma){ }^{16} \mathrm{O}$ rate. Another explanation in the case of $\mathrm{S} / \mathrm{O}$ and $\mathrm{Ar} / \mathrm{O}$ may be that the predicted yields do not include contributions from Type Ia supernovae, which produce significant amounts of ${ }^{32} \mathrm{~S}$ and ${ }^{36} \mathrm{Ar}$, according to Nomoto et al.'s (1997a) W7 model. Adding this source to yields of massive stars would raise the theoretical line. This subject should be explored in more detail, particularly since the $\mathrm{Ne} / \mathrm{O}$ ratio ought to provide a good constraint on the value of the ${ }^{12} \mathrm{C}(\alpha, \gamma){ }^{16} \mathrm{O}$ rate. 


\section{Abundance Patterns In Galaxies From Stars}

Stars are substantially fainter than H II regions, planetary nebulae, or supernova remnants in general, and therefore can only be observed one by one in the Milky Way except for the brightest giants and supergiants, some of which can be observed out to about $10 \mathrm{Mpc}$. Additionally, abundance measurements in stars come from relatively precise measurements of the depth of absorption features (rather than emission features), therefore requiring more photons for results of similar accuracy. Furthermore, it is usually the more inconspicuous lines from which the most reliable abundances are derived! However, stars have one distinct astrophysical advantage: they are long-lived. Study of stars of different ages can reveal chemical history explicitly, rather than implicitly through chemical evolution models. In this section we briefly survey the extant stellar results for the MWG and external galaxies. A brief description of how abundances are inferred from stellar spectra is provided in Appendix B.

\subsection{Milky Way Galaxy}

To summarize four decades of work on Milky Way stellar abundances in a balanced fashion is clearly beyond the scope of a single paper, so we attempt to provide an executive summary. Workers now subdivide the Milky Way into the spheroidal halo ( $\mathrm{r}>2 \mathrm{kpc}$ ) and bulge $(\mathrm{r}<2 \mathrm{kpc})$ and two disk-like components, the thick disk (scale height $\approx 1 \mathrm{kpc}$ ) and the thin disk (scale height $\approx 350 \mathrm{pc}$ ). We now treat these in order.

The halo has a metal abundance of $[\mathrm{Fe} / \mathrm{H}] \approx-1.6 \pm 1$ w with no noticeable abundance gradient. This information comes from studies of individual subdwarfs (Carney et al. 1990, 1996). Globular clusters fall into two spatially and kinematically distinct groups; the inner, metal-rich disk clusters and the outer, metal-poor halo clusters. Internal to either group there is no clear abundance gradient (Zinn 1996; Richer et al. 1996). Increasing evidence suggests that the globular cluster system has a significant age spread of 3-4 Gyr (e.g. Hesser et al. 1997), which may depend on radius; older toward the center. There is also a pattern of lighter species becoming enhanced relative to Fe-peak elements in stars more metal-poor than about $[\mathrm{Fe} / \mathrm{H}]=-1$, with $\mathrm{O}, \mathrm{Mg}, \mathrm{Al}, \mathrm{Si}, \mathrm{Ca}$, and $\mathrm{Ti}$ overabundant by several tenths of a dex relative to a scaled-solar mixture in the metal-poor group (Wheeler et al. 1989; Edvardsson et al. 1993). The standard interpretation is that the metal-poor group was

\footnotetext{
${ }^{5}$ Here we employ the standard bracket notation often used in expressing abundances, $[\mathrm{X}] \equiv \log (\mathrm{X})-$ $\log (\mathrm{X}) \odot$, where $\mathrm{X}$ represents an elemental abundance or an abundance ratio. We will use the symbol $\mathrm{Z}$ to represent all heavy elements at once.
} 
enriched mainly by Type II supernova nucleosynthesis products and the metal-rich stars contain a mixture of Type II and Type I products, where the Type I supernovae are thought to produce mainly Fe-peak elements.

The Galactic bulge has much foreground dust as well as confusion with foreground disk stars and thus is a difficult place for observational work. Photometric studies seem to indicate a negative abundance gradient but the size of the gradient is not yet well-quantified (Terndrup 1988; Frogel et al. 1990; Harding 1996). Due to the reddening, spectroscopy seems like a safer way to proceed. Still, results are ambiguous. Ibata \& Gilmore (1995) find a near-solar metallicity but no gradient outwards from $0.6 \mathrm{kpc}$, but Terndrup et al. (1990) and Rich (1998) find a gradient of about $-0.4 \mathrm{dex} / \mathrm{kpc}($ or $\Delta \log (\mathrm{Z}) / \Delta \log \mathrm{R} \approx-0.6$ ) considering regions somewhat further toward the Galactic center.

The thick disk, massing about $10 \%$ of the thin disk, is separable from the thin disk and halo primarily through kinematics or age, since its metallicity overlaps at the high end with the thin disk (Wyse \& Gilmore 1995) and at the low end with the halo (Nissen \& Schuster 1997). No radial or vertical gradient in $[\mathrm{Fe} / \mathrm{H}]$ has been discovered in several large data sets (Gilmore et al. 1995; Bell 1996; Robin et al. 1996). The high-quality data of Edvardsson et al. (1993) confirms the lack of a strong gradient for heavy elements, but finds a probable relation between "alpha" elements $\mathrm{Si}$ and Ca relative to Fe as a function of radius over a 4 - to $12-\mathrm{kpc}$ span of about $[\alpha / \mathrm{Fe}] / \mathrm{R}_{\mathrm{m}}=+0.03 \mathrm{dex} / \mathrm{kpc}$, where $\mathrm{R}_{\mathrm{m}}$ is an estimate of the radius at which the stars were born, rather than where they are presently located. Similarly, $[\alpha / \mathrm{Fe}]$ has been found to increase with age. Small amplitude results of this nature should become more common as stellar abundances become more accurate.

The thin disk can be traced by open clusters, most of which are younger than about half the age of the disk. Friel \& Janes (1993) and Thogersen et al. (1993) worked with moderate resolution spectra of open cluster $\mathrm{K}$ giants to obtain a mean $[\mathrm{Fe} / \mathrm{H}]$ gradient of $-0.097 \pm 0.017 \mathrm{dex} / \mathrm{kpc}$ between 7 and $15 \mathrm{kpc}$. Photometry of open cluster stars has yielded similar results (Panagia \& Tosi 1981; Cameron 1985). An alternative to a steady gradient has been proposed by Twarog et al. (1997), who from a sample of 76 open clusters find a sharp falloff of roughly 0.35 dex at around $10 \mathrm{kpc}$ from the Galactic center with flat gradients interior and exterior to that radius.

Of similar luminosity to $\mathrm{K}$ giants are B main sequence stars. B stars are youthful in age, so their abundances should match those of H II regions. Accurate spectral analysis of oxygen lines in (at least) the hotter B stars is dependent on dropping the assumption of "local thermodynamic equilibrium" between the radiation field and the gas. Smartt \& Rolleston (1997) and Gummersbach et al. (1998) have undertaken such an analysis, deriving an $[\mathrm{O} / \mathrm{H}]$ gradient of $-0.07 \pm 0.01 \mathrm{dex} / \mathrm{kpc}$, a result different from most of its 
predecessors, but in agreement with the nebular results.

Figure 9 shows a summary of the broad-brush abundance pattern in the Milky Way. The metal-poor halo weighs about $10^{9} \mathrm{M}_{\odot}$ (e.g. Freeman 1996) compared to about $60 \times 10^{9} \mathrm{M}_{\odot}$ for the total mass (in stars) in the Galaxy, so the number of symbols on the plot does not reflect where the mass is; most of the mass resides in the disk. Two trends are evident from the figure: abundance increases with time, and the abundance is higher toward the Galactic center. Coupled with stellar kinematics and age information, these abundances give a picture of Galaxy formation in which the halo formed early and without much chemical enrichment. The disk may have started early as well, but it is still gas rich and is still forming stars today at near-solar abundance.

\subsection{External Galaxies}

Spectroscopy of individual stars in local group galaxies M31 and M33 has become possible in recent years for supergiants, typically A-type. Like B main sequence stars, A supergiants suffer from serious non-LTE effects in the outer photosphere, but lines can be chosen that form deep in the photosphere and a partial non-LTE analysis can be attempted for other interesting lines. The resultant accuracy can be $\pm 0.2 \operatorname{dex}$ (Venn 1995; 1998). For $\mathrm{M} 31$, the $[\mathrm{O} / \mathrm{H}]$ gradient obtained from A supergiants is consistent within the errors with that obtained from nebular studies (McCarthy et al. 1998). For M33, based on four B supergiants, Monteverde et al. (1997) obtain an $[\mathrm{O} / \mathrm{H}]$ gradient of $-0.16 \pm 0.06 \mathrm{dex} / \mathrm{kpc}$, which is also similar to nebular results.

Most stellar abundance work in external galaxies relies on the colors of red giant stars from older populations. After spectroscopic abundance work in globular clusters showed a wide range of metallicities among clusters, it was obvious from published color-magnitude diagrams (CMDs) that the red giant branches are redder at progressively higher metallicities. This finding can be used as an abundance indicator, especially in the HST era where the tip of the red giant branch can be seen at distances of $\sim 10 \mathrm{Mpc}$. A younger age population has a somewhat bluer giant branch, but this effect is fairly minimal, and in some cases negligible when the age is already known. Crowding of stars excludes near-nuclear regions from CMD analysis.

The halo of M31 has been examined by Durrell et al. (1994) and Rich et al. (1996) from HST optical colors, with the conclusion that, like the Milky Way, no abundance gradient is apparent. Unlike the Milky Way, the average abundance of the stars is $[\mathrm{Fe} / \mathrm{H}]$ $\approx-0.6$ (Durrell et al.) or even higher (Rich et al.). Grillmair et al. (1996) derive an 
abundance distribution (number of stars per interval $[\mathrm{Fe} / \mathrm{H}]$ ) for the outer disk of M31 that is identical within the errors with the abundance distribution of the solar neighborhood. Elliptical galaxies NGC 5128 (Soria et al. 1996) and M32 (Grillmair et al. 1996) have also been studied in this fashion, but only at a single radius so far, so we await further data before we can draw conclusions about abundance profiles.

\section{Abundance Patterns In Spheroidal Systems From Photometric Observations}

\subsection{Metallicity Gradients}

We now provide an overview of the abundance profile picture for spheroidal systems, especially elliptical galaxies. The principal techniques for measuring abundances in these systems involve the use of photometric indices of integrated starlight, since individual stars cannot be resolved. A brief description of these techniques is given in Appendix C. Elliptical galaxies look superficially like a fairly homogeneous class of galaxies, with muted star formation and no obvious cold gas, and kinematically supported by almost randomly oriented orbits. Star formation can be seen in most ellipticals at some level, as can dust lanes and emission-line gas, but usually at a level below that of spirals. Ellipticals also exhibit regularity of colors and absorption feature strengths, with larger galaxies being redder and having stronger metallic absorption features than smaller ones. This has long been interpreted as a sign that the metallicity is higher in larger galaxies (e.g. Faber 1972).

To derive abundance profiles in stellar systems, colors and absorption feature strengths as a function of galactocentric radius are interpreted through population models. Some color gradient studies include Kormendy and Djorgovski (1989), Franx \& Illingworth (1990), and Peletier et al. (1990). Most studies of optical absorption features have utilized one particular system of feature indices developed at Lick Observatory (described in Worthey et al. 1994 and references therein). The last few years have seen a rapid expansion of galaxy data available in this system. To measure an absorption feature in the Lick system, one creates a pseudocontinuum by bracketing the spectral feature of interest with flanking passbands. Flux in the flanking bands is measured and a straight line is drawn between the midpoints of the flanking bands to represent the (pseudo)continuum. The flux difference between the pseudocontinuum and the absorption feature is integrated and the result is expressed in $\AA$ of equivalent width (or magnitudes, depending on the specific index; see Worthey et al. 1994 and Worthey \& Ottaviani 1997 for the details.) Figure 10 illustrates the idea for a portion of the spectrum. There are 25 indices defined, 5 definitions measuring Balmer lines and 20 measuring various metallic absorption blends. The index system 
operates at a low resolution $(\sim 8 \AA \mathrm{FWHM})$ necessitated by Doppler smearing from the substantial (up to $\sigma=350 \mathrm{~km} / \mathrm{s}$ ) velocity dispersions of large elliptical galaxies, and most of the indices require corrections when velocity dispersions get large.

While most of these 25 indices follow the $\Delta \log ($ Age $) / \Delta \log \mathrm{Z} \approx-\frac{3}{2}$ constant-index slope described in Appendix $\mathrm{C}$, a few (the Balmer indices) are relatively age sensitive, with $\Delta \log ($ Age $) / \Delta \log \mathrm{Z} \approx-\frac{1 \text { to } 2}{2}$, while others, notably a feature called Fe4668 whose main contributor is really molecular carbon, are relatively metal sensitive, with $\Delta \log ($ Age $) / \Delta \log \mathrm{Z} \approx-5$ (Worthey 1994). Arrayed against each other, it seems possible to separate the effects of age and metallicity, in the mean.

To derive an abundance gradient in an elliptical galaxy, one compares observed colors or line strengths with model predictions, often assuming a constant age throughout the galaxy. For instance, Franx \& Illingworth (1990) find a mean color gradient in 17 elliptical galaxies of $\Delta(\mathrm{U}-\mathrm{R}) / \Delta \log \mathrm{r}=-0.23 \pm 0.03 \mathrm{mag}$ per decade in radius. Entering the Worthey (1994) models at age $12 \mathrm{Gyr}$, one finds that a change of 0.15 dex in $\mathrm{Z}$ gives the required $\Delta(\mathrm{U}-\mathrm{R})$, so the gradient assuming constant age is $\Delta \log \mathrm{Z} / \Delta \log \mathrm{R}=-0.15$ dex per decade. The same number is reached by considering the $\mathrm{B}-\mathrm{R}$ gradient. Due to the very steep surface brightness dropoff of elliptical galaxies, projection effects are small and usually neglected. The steep dropoff also means that long-slit spectroscopy usually only reaches to 0.5 to $1.0 \mathrm{R}_{\mathrm{e}}$ (the half-light radius) although color gradient studies and ultradeep spectroscopy can reach to several $R_{e}$.

Color studies and line strength studies generally give a consistent picture of a gradient of about $\Delta \log \mathrm{Z} / \Delta \log \mathrm{R} \approx-0.2$ dex per decade. There is probably a small correction to this number, however, due to age effects. Simultaneous mean-age, mean-Z estimates using the Balmer-versus-metal feature technique described above were derived for the González (1993) and Mehlert et al. (1998) samples of galaxies, about 60 early type galaxies in a wide variety of environments, and the distribution of gradients is shown in Fig. 11a. There are no trends of gradient strength with luminosity or velocity dispersion (unlike average Z, which is larger in larger galaxies). The average age gradient is younger toward the center by $0.1 \mathrm{dex} /$ decade (a few Gyrs), and more metal-rich by $0.25 \mathrm{dex} /$ decade. The scatter in the average seems mostly due to observational error, error in correcting for $\mathrm{H} \beta$ emission fill-in, and variation in abundance ratio mixture, and the residuals scatter along the $-\frac{3}{2}$ age-metallicity slope (Fig. 11b) in the way expected for random errors in input index values.

This -0.3 gradient in dex/decade units corresponds to about $-0.02 \mathrm{dex} / \mathrm{kpc}$ assuming an $8 \mathrm{kpc}$ radius, which is a factor of three more shallow than the gradient found for the Milky Way disk and other non-barred spirals (see $§ 2.1$ ). But such a value is well within the 
range of theoretical models for galaxy formation. For example, Larson's (1974) dissipative models predict $\Delta \log \mathrm{Z} / \Delta \log \mathrm{R}=-1$, while various Carlberg (1984) models range from -0.5 to 0.0. Pure stellar merging gives zero gradient, and in fact tends to erase pre-existing gradients by roughly $20 \%$ per event, or even more via changes in radial structure of the galaxies (White 1980).

The gradient numbers seem fairly robust and consistent from data set to data set and from model to model. What about absolute abundances? These are trickier. The nuclei of large elliptical galaxies have mean $[\mathrm{Z} / \mathrm{H}]$ in the range 0.0 to 0.4 dex as inferred from Balmer-versus-metal feature diagrams. In principle, the mean abundance can be known much more precisely, but there is an additional stumbling block beyond just the inaccurate models and the complication of age-metal degeneracy. The elemental mixture in elliptical galaxies is not scaled-solar. Abundances derived from lighter-element lines like $\mathrm{Mg} \mathrm{b}$ or $\mathrm{Na}$ $\mathrm{D}$ are much higher than those derived from heavier Fe or Ca lines, and this is the main cause for uncertainty in the absolute abundance (Worthey 1998).

\subsection{Enhanced Light-to-Heavy Element Ratios}

The light elemento enhancement can be seen in the case of $[\mathrm{Mg} / \mathrm{Fe}]$ by plotting a magnesium feature index $\left(\mathrm{Mg}_{2}\right)$ versus an average iron feature $(<\mathrm{Fe}>=$ the arithmetic average of indices Fe5270 and Fe5335). The age sensitivities of these indices are about the same $\left(\approx \frac{3}{2}\right.$ !), so models of different ages and metallicities should lie on top of one another. They do, as seen in Figure 12, but at high Mg strength the galaxies follow another distinct trajectory entirely with some galaxies trending toward strong $\mathrm{Mg}_{2}$ strength at nearly constant $<\mathrm{Fe}>$, and hence, we infer, relatively enhanced $\mathrm{Mg}$ abundance.

The models are scaled-solar since they are built from local stars, so they cannot track altered abundances. Composite populations add approximately like vectors, so any combination of ages and metallicities still lands on the same model locus. Different models built by different authors have a spread of something like \pm 0.5 dex at constant index strength, but all models follow almost exactly the same slope in the Fig. 12 diagrams, so that the inferred $[\mathrm{Mg} / \mathrm{Fe}]$ for the high- $\mathrm{Mg}_{2}$ group of elliptical galaxies is in the range $[\mathrm{Mg} / \mathrm{Fe}]=0.3$ to $0.5 \mathrm{dex}$.

\footnotetext{
${ }^{6}$ In this subsection we make a distinction between "light" and "heavy" elements, divided at the fourth row of the periodic table, so that $\mathrm{Ca}$ and $\mathrm{Fe}$ are heavy, but $\mathrm{Na}, \mathrm{Mg}$, and $\mathrm{N}$ are light. The oft-standard terminology is to speak of "alpha" elements, but "alpha" usually includes Ca and excludes N, which makes little sense for the abundance pattern seen in massive elliptical galaxies.
} 
A crucial thing to notice is that velocity dispersion tracks $\mathrm{Mg}_{2}$ very tightly, so the high $\mathrm{Mg}_{2}$ galaxies are also the largest galaxies (or, more precisely, the "dynamically hottest"). The $\mathrm{Mg}_{2}-\sigma$ relation is shown in Figure 13; it is one of the tighter scaling relations known, much tighter than, say, the $<\mathrm{Fe}>-\sigma$ diagram, which is almost a scatter plot. With the existence of the $\mathrm{Mg}-\sigma$ relation, $[\mathrm{Mg} / \mathrm{Fe}]$ increases with galaxy size, where a velocity dispersion of $\sigma \approx 200 \mathrm{~km} / \mathrm{s}$ seems to mark the beginning of noticeable $\mathrm{Mg}$ enhancement.

Figure 12 shows separate diagrams for spiral bulges, S0 galaxies, and elliptical galaxies. No marked difference between Hubble types is seen except that already ascribed to velocity dispersion. That is, spiral bulges hover near the solar ratio area, but only two bulges have $\sigma>200 \mathrm{~km} / \mathrm{s}$ (and those are on the high-Mg side of the distribution). Elliptical galaxies possess both the most extreme velocity dispersions and the most extreme $\mathrm{Mg}$ enhancement.

The gradient vectors shown in Figure 12 tend to parallel the age-metallicity direction traced by the various models rather than the more horizontal slope defined by the nuclei. This would suggest that the Mg enhancement is global throughout the galaxy rather than concentrated only at the nucleus. Is this a hint that the enrichment mechanism (presumably supernova) spreads enriched gas 10 or $20 \mathrm{kpc}$ from its origin, or does it merely imply effective mixing?

The $[\mathrm{Mg} / \mathrm{Fe}]$ data suggest a variation in enrichment from Type Ia (mostly Fe) and Type II (all elements) supernovae passing from small galaxies or bulges to large ones, in the sense that the large galaxies have more $\mathrm{Mg}$ and hence comparatively more Type II

enrichment. Figure 14 shows some corroborating evidence from Trager et al. (1998) nuclear data in that $\mathrm{Ca}$ appears to track $\mathrm{Fe}$, while $\mathrm{Na}$ and $\mathrm{N}$ are enhanced in a way similar to that of $\mathrm{Mg}$; only in the larger galaxies or bulges.

The mechanism for modulating Type I/Type II enrichment is not known. It could be due to a time delay in Type I metal production, or could be some other connection to velocity dispersion like a variable IMF or a variable fraction of binary stars. (Worthey, Faber, \& González 1992; Weiss, Peletier, \& Matteucci 1995).

\subsection{The G Dwarf Problem: a peaked abundance distribution}

The abundance distribution (number of stars versus $[\mathrm{Fe} / \mathrm{H}]$ ) in the Milky Way galaxy is more strongly peaked than the simplest closed-box model (see §1) with constant yield predicts. This is known as the G dwarf problem (van den Bergh 1962; Pagel 1997, Cowley 1995). It seems almost certain now that other galaxies share this "problem" of having a relatively peaked abundance distribution. Part of the evidence comes from integrated light 
(Bressan et al. 1994; Worthey, Dorman, \& Jones 1996) via three lines of evidence.

First, around $2600 \AA$ there is a paucity of ultraviolet flux which would otherwise be greater if large numbers of metal-poor main sequence stars are present. Second, in small compact ellipticals for which data exist, a high-resolution Ca II index (Rose 1994 system, not Lick system) detects few A-type horizontal branch stars, where more of these objects would be expected if a large metal-poor population exists. Third, if a metal-sensitive index like Fe4668 is modeled using the broad simple model predictions, the strong line strengths in large galaxies are very difficult to attain, and require improbably high yield values. Clinching the integrated light results, recent color-magnitude diagram studies of individual red giants in the compact elliptical M32 (Grillmair et al. 1996), large elliptical NGC 5128 (Soria et al. 1996), and the disk of M31 (Grillmair et al. 1996) all indicate a very peaked abundance distribution similar to or more peaked than that of the Milky Way.

These empirical findings represent important constraints on some galaxy formation and chemical evolution models, and their implications are only starting to be explored (Larson 1998).

\subsection{Assorted Issues}

The $M g_{2}-\sigma$ relation (Fig. 13) is tighter than other population-to-structural correlations such as $\mathrm{Mg}_{2}-\mathrm{M}_{\mathrm{B}}$, or $<\mathrm{Fe}>-\sigma$. (Bender, Burstein, Faber 1993) There is some powerful connection between velocity dispersion and $\mathrm{Mg}$ abundance as traced by the $\mathrm{Mg}$ b feature, the exact nature of which eludes us at the moment. One possibility, suggested by Faber et al. (1992), is that cloud-cloud collision velocity modulates the IMF to favor more massive stars in high- $\sigma$ environments. This suggestion is in harmony with the $\mathrm{Mg} / \mathrm{Fe}$ abundance trend. Another possibility is that larger local escape velocities resist supernova winds more effectively, holding onto heavy-element contaminants better. Star formation is finally truncated when supernova winds are able to blow the remaining gas out of the galaxy. This is the now-standard picture of chemical evolution in elliptical galaxies (e.g. Arimoto \& Yoshii 1987, Matteucci \& Tornambé 1987). This picture can also be made harmonious with the $\mathrm{Mg} / \mathrm{Fe}$ trend if there is an additional mechanism for varying $\mathrm{Mg} / \mathrm{Fe}$ as a function of

galaxy size, but the fact that Fe abundance is virtually independent of galaxy size causes some trouble.

Discontinuities in kinematic profiles are coincident with discontinuities in line strength profiles. Bender and Surma (1993) discovered in the course of studying peculiar kinematics in elliptical galaxies that many have counter-rotating cores or other kinematic 
discontinuities. In every case that they studied, a discontinuity appeared in the $\mathrm{Mg}_{2}$ line strength profile at the same location as the kinematical discontinuity. At the very least, this implies that whatever formation mechanism produced the distinct core also influenced the local chemistry. Muted echoes of formation exist still in both the stellar kinematics and the chemical signature in the stars.

Study of the globular cluster systems around elliptical galaxies yields insight into the formation of halos in general and elliptical galaxies in particular. Some elliptical galaxies host a very large number of globular clusters per unit luminosity (e.g. M87) while others have about as many as are seen in spiral galaxies. Questions remain about how the globular clusters are created and destroyed to explain the wide variation in number and whether merging events are important or not (van den Bergh 1995; Zepf \& Ashman 1993). Most abundance studies concentrate on the integrated colors of the clusters (Ostrov et al. 1998; Lee et al. 1998; Ajhar et al. 1994) because of their faintness, but some spectroscopic studies are beginning to appear (e.g. Cohen et al. 1998). These efforts show a variety of interesting results. For example, some globular cluster systems are metal-poor, some metal-rich, and some bimodal or multimodal. And when both metal-poor (blue) and metal-rich (red) populations coexist, the red population tends to be more centrally concentrated than the blue.

\section{Summary and Suggestions}

We have explored in some detail the abundance patterns in spiral disks and elliptical galaxies as revealed through analyses of gaseous nebulae, stars and integrated photometry of galaxies.

The principal points regarding abundance patterns in spiral disks are:

- The metallicity as gauged by $\mathrm{O} / \mathrm{H}$ in nebulae across the Milky Way disk decreases with galactocentric distance, a finding supported by recent abundance results for disk stars. This negative gradient pattern is seen in most other spiral disks. A similar result is seen when luminous stars are used as abundance probes. Scatter at any particular galactocentric distance is consistent with observational uncertainty.

- Global metallcity, taken as the abundance of oxygen at a standard galactocentric distance, is positively correlated with galaxy mass.

- Metallicity at any location in a spiral disk appears to be positively correlated with the local total surface density. 
- Abundance gradients are steeper in normal spirals than in barred ones.

- A plot of $\mathrm{N} / \mathrm{O}$ versus $\mathrm{O} / \mathrm{H}$ in spiral disks indicates that production of nitrogen is dominated by primary processes at low metallicities and secondary processes at high metallicities.

- $\mathrm{C} / \mathrm{O}$ is positively correlated with $\mathrm{O} / \mathrm{H}$ in spiral disks, suggesting that carbon production is sensitive to metallicity, possibly through metallicity-enhanced mass loss in massive stars.

- Abundance ratios of $\mathrm{Ne} / \mathrm{O}, \mathrm{S} / \mathrm{O}$, and $\mathrm{Ar} / \mathrm{O}$ appear to be universally constant across the range in metallicities observed, reflecting the idea that either the initial mass function is universally constant; or the stellar mass range responsible for producing these elements is relatively narrow, and thus these ratios are insensitive to IMF variations.

- Stellar age and galactocentric distance in the Milky Way show rough correlations with metallicity in the sense that metallicity decreases with increasing age and galactocentric distance. However, all Galactic components (halo, bulge, thin disk, thick disk) have large scatter in abundance, and even the metal-poor halo is now thought to display age scatter of several Gyr.

For elliptical galaxies, the main results are:

- Abundance gradients are, on average, about a factor of two to three more shallow than in non-barred spirals. This is well within the range expected from various formation pictures, including hierarchical mergers of smaller galaxies.

- Nuclear or global metallic feature strengths (or colors) become stronger (or redder) in larger galaxies. The 1970's conclusion that larger elliptical galaxies must be more metal-rich is reconfirmed, but every elemental species does not increase in lockstep.

- Light elements $\mathrm{N}, \mathrm{Na}$, and $\mathrm{Mg}$ are enhanced relative to heavy elements $\mathrm{Ca}$ and $\mathrm{Fe}$ in the largest elliptical galaxies, implying a modulation of enrichment, plausibly due to variance of the Type II to Type Ia supernova ejecta, compared to smaller ellipticals, bulges, and disks.

- The mean abundance near the nuclei of large elliptical and S0 galaxies is uncertain, but is in the range $[\mathrm{Z} / \mathrm{H}]=0.0$ to 0.4 . Most of the difference in abundance between small and large galaxies is driven by the increasing abundance of elements lighter than 
those near the Fe-peak, with $[\mathrm{Fe} / \mathrm{H}]$ staying roughly constant for elliptical galaxies of all sizes.

- The abundance distribution in elliptical galaxies and, so far, every other well-studied large galaxy type, is strongly peaked like that of the solar cylinder, not broad like the simplest closed-box model predicts.

There are two over-arching patterns which emerge from the combined results for spirals and ellipticals. First, there is a positive correlation between galactic metallicity and mass. This may be related to the greater retension of heavy elements ejected by supernovae by the stronger gravitational potentials of massive galaxies, or perhaps to the effects of galaxy mass on the star formation process. It is currently difficult to ascertain whether this relation is completely continuous across galaxy types; in other words, if one plotted global metallicity versus mass for a sample of galaxies containing both spirals and ellipticals would there be an unbroken straight line, or would the correlation for one type be offset from the other. The difficulty here is in directly comparing abundances between the two galaxy types. As we have seen, metallicity in spirals is generally gauged by observing oxygen in nebulae located in their disks. Yet in ellipticals, metallicity must be measured from integrated light using numerous photometric indices which are affected not only by metallicity but by age. Thus, no seamless technique exists for determining abundances consistently for spirals and ellipticals, and thus intercomparisons are problematic. This is made all the more complicated by the fact that we currently don't know how to represent the global abundance in a galaxy. Do we take the abundance at the nucleus, or at one effective radius, or at 0.4 optical radii?

The second pattern which has emerged is that abundance gradients appear to become flatter as one progresses from normal spirals to barred spirals to ellipticals. The difference between normal and barred spirals is currently explained by enhanced radial gas flows in the disks of barred spirals. To extend this model to ellipticals it may be neccessary to invoke other radial mixing mechanisms, either during primordial formation or during later merging events. If the pattern is discontinuous between spirals and ellipticals this might suggest that different processes operate to affect the gradients in the two galaxy types. Again, our lack of ability to intercompare spiral and elliptical abundances prevents further exploration of this pattern at present.

Understanding the broad picture of galactic chemical evolution will require us to firm up the links between elliptical and spiral galaxy abundances. While a common elemental yardstick may not exist because of the different elements which we observe directly in each galaxy type, it may be possible to tie the two types together abundance-wise by observing 
elements in each which share the same production site nucleosynthetically speaking. An example might be oxygen and magnesium. In external spirals oxygen is taken as the metallicity gauge primarily because of its observability. Magnesium, which, like oxygen, is primarily produced in massive stars (Nomoto et al. 1997a,b) may be measurable directly through a calibrated $\mathrm{Mg}_{2}$ index. Then oxygen and magnesium might be linked by assuming a "cosmic" $\mathrm{Mg} / \mathrm{O}$ ratio calibrated locally. Also, although the work is not started, it may be possible to construct an oxygen-sensitive photometric index for integrated light, perhaps revolving around the $2.3 \mu \mathrm{m} \mathrm{CO}$ feature in conjunction with the $\mathrm{C}_{2}$-sensitive 4668 feature. As synthetic spectra and stellar abundances grow more precise, these speculative suggestions might take place, leading to a much more clear understanding of chemical enrichment and galaxy formation.

This collaboration was inspired by the October, 1997, workshop "Abundance Profiles: Diagnostic Tools For Galaxy History" held at Université Laval, Québec. We are grateful to the organizers for giving all of us the opportunity to come together and share our ideas about what one participant, in an attempt to relabel the abundance field with a trendier and more attention-grabbing name, referred to as "bio-resources". We also thank Bill Blair, César Esteban, Mike Fich, George Jacoby, Yuri Izotov, Joachim Köppen, Walter Maciel, Dörte Mehlert, Anne Sansom, Jan Simpson, and Friedl Thielemann for promptly responding to inquiries with useful answers and information given generously. And finally, we are especially grateful to our referees, Dave Burstein, Karen Kwitter, and Bernard Pagel, for promptly and carefully reading the manuscript and making numerous constructive comments which have improved the paper tremendously.

\section{Appendix A}

\section{Abundances From Emission Line Objects}

We present here only a brief overview of abundance determining methods relevant to emission-line objects. A list of prominent nebular emission lines is presented in Table A1, where we provide the ion, wavelength, and dominent excitation mechanism for each line. Readers interested in greater detail are urged to consult information in Spitzer (1978), Aller (1984), Osterbrock (1988; 1989), and Williams \& Livio (1995). The atomic data used for the abundance calculations are reviewed by Butler (1993).

The basic method for obtaining the abundance of an element in emission-line objects comprises two steps: (1) determine abundances of the ions of that element whose emission 
lines are directly observable; and (2) adjust the total of the ionic abundances by a factor which accounts for ions of the same element which are unobservable.

Consider step 1. An ionic abundance relative to $\mathrm{H}^{+}$is related to the observed strength of an emission line integrated over wavelength, corrected for interstellar reddening, and expressed relative to the $\mathrm{H} \beta$ strength, $\mathrm{I}_{\lambda} / \mathrm{I}_{\mathrm{H} \beta}$, through the respective reaction rate coefficients, $\epsilon_{\lambda}$ and $\epsilon_{\mathrm{H} \beta}$ in $\operatorname{erg} \mathrm{cm}^{3} \mathrm{~s}^{-1} \mathrm{sr}^{-1}$, such that:

$$
\frac{\mathrm{I}_{\lambda}}{\mathrm{I}_{\mathrm{H} \beta}}=\frac{\int \epsilon_{\lambda}\left(\mathrm{T}_{\mathrm{e}}, \mathrm{N}_{\mathrm{e}}\right) \mathrm{N}_{\mathrm{i}} \mathrm{N}_{\mathrm{e}} \mathrm{ds}}{\int \epsilon_{\mathrm{H} \beta}\left(\mathrm{T}_{\mathrm{e}}, \mathrm{N}_{\mathrm{e}}\right) \mathrm{N}_{\mathrm{H}^{+}} \mathrm{N}_{\mathrm{e}} \mathrm{ds}}
$$

where $\mathrm{N}_{\mathrm{i}}, \mathrm{N}_{\mathrm{H}^{+}}$, and $\mathrm{N}_{\mathrm{e}}$ are local number densities of the ion giving rise to the line $\lambda, \mathrm{H}^{+}$, and electron density. The integrals arise because local products are integrated along the line of sight. Note that the rates are functions of the local electron temperature $\left(\mathrm{T}_{\mathrm{e}}\right)$ and density $\left(\mathrm{N}_{\mathrm{e}}\right)$, although a good simplifying assumption is that these are constant within the nebular regions actually dominated by the ions in question. $\mathrm{T}_{\mathrm{e}}$ is usually determined using the line strength ratio of two lines such as [O III] $\lambda 4363$ and $\lambda 5007$ whose upper energy levels are relatively far apart. $\mathrm{N}_{\mathrm{e}}$ is derived from the ratio of two lines such as [S II] $\lambda 6716$ and $\lambda 6731$ whose upper energy levels are closely spaced but the transitions differ significantly in their sensitivities to collisional deexcitation.

Adding the observed ionic abundances for an element together gives us a subtotal which differs from the desired total by the abundances of the ions whose emission lines are not observed. Thus, in step 2 above we determine an ionization correction factor, icf(X), for element $\mathrm{X}$ by which we multiply the subtotal to produce the total elemental abundance relative to $\mathrm{H}^{+}$. Mathematically, the number density of an element $\mathrm{N}_{\mathrm{X}} / \mathrm{N}_{\mathrm{H}}$ can be expressed as:

$$
\frac{\mathrm{N}_{\mathrm{X}}}{\mathrm{N}_{\mathrm{H}^{+}}}=\left\{\sum^{\text {obs }} \frac{\mathrm{N}_{\mathrm{i}}}{\mathrm{N}_{\mathrm{H}^{+}}}\right\} \cdot \operatorname{icf}(\mathrm{X} / \mathrm{H}) .
$$

Ionization correction factors may be inferred from model simulations of nebulae or estimated by assuming that ions with similar ionization potentials are present in the gas in similar ratios to their total abundances. Thus, because the ionization potentials of $\mathrm{O}^{+2}$ and $\mathrm{He}^{+}$ are $54.9 \mathrm{ev}$ and $54.4 \mathrm{ev}$, respectively, the total abundance of unobservable (in the optical) higher ionization stages such as $\mathrm{O}^{+3}$ and beyond with respect to total $\mathrm{O}$ is similar to relative amounts of $\mathrm{He}^{+2}$, an optically observable ion, with respect to total He. A good compilation and discussion of a broad range of icf's may be found in the appendix of Kingsburgh \& Barlow (1994). Additionally, model grids such as those by Stasińska \& Schaerer (1997) may be used to derive icf's for a nebula assuming the central star temperature is known.

The above method breaks down most frequently for metal-rich nebulae with low equilibrium temperatures, in which case auroral lines such as [O III] $\lambda 4363$ are too weak to 
measure, and thus the temperature cannot be determined. One way around the problem is to calculate a detailed photoionization model of the nebula using input abundances and other physical parameters which produce an output set of line strengths closely matching the observed ones. Actual abundances are then inferred from the model input. A simpler solution is the "strong-line method", which uses a composite of strong, observable emission line strengths whose value tracks an abundance ratio. The most significant example is $\mathrm{R}_{23} \equiv([\mathrm{O}$ III $]+[\mathrm{O}$ II $]) / \mathrm{H} \beta$, first introduced by Pagel et al. (1979), which comprises the sum of $[\mathrm{O}$ III] and [O II] nebular line strengths relative to the strength of $\mathrm{H} \beta$ and is related in a complicated but understandable way to the total oxygen abundance $\mathrm{O} / \mathrm{H}$. This method has been refined and discussed more recently by Edmunds \& Pagel (1984), Edmunds (1989) and McGaugh (1991), and of course it is not problem-free. Because the metal-rich portion of this relation must currently be calibrated with models, uncertainties arise from parameter choices such as relative depletion (Henry 1993; Shields \& Kennicutt 1995) and gas density (Oey \& Kennicutt 1993). Finally, analogous methods for obtaining N/O and S/O are presented by Thurston, Edmunds, \& Henry (1997) and Díaz (1999), respectively.

Finally, the accuracy of abundances in emission-line systems is threatened by the proposed existence of small scale temperature fluctuations along the line-of-sight, first described by Peimbert (1967). In this picture, an electron temperature measured with forbidden lines is actually overestimated when fluctuations are present but ignored. This in turn causes an underestimation of an abundance ratio such as $\mathrm{O}^{+2} / \mathrm{H}^{+}$when it's based upon a forbidden/permitted line ratio such as [O III $] \lambda 5007 / \mathrm{H} \beta$. When ratios of permitted lines are used the effect is minimal and so abundances inferred from permitted/permitted line ratios are unaffected and systematically higher than abundances from forbidden/permitted ratios. Temperature fluctuations have been used to explain, among many other things, the significant discrepancy in planetary nebula carbon abundances (Peimbert, Torres-Peimbert, \& Luridiana 1995), where those determined using C II $\lambda 4267 / \mathrm{H} \beta$, say, are often several times greater than abundances inferred from C III] $\lambda 1909 / \mathrm{H} \beta$. Esteban et al. (1998) found the effect to be small in the Orion Nebula, while Liu (1998) found a large effect in the planetary nebula NGC 4361, although it was insufficient for explaining the discrepancy between carbon abundances from recombination and collisionally excited lines. The issue of temperature fluctuations is an important one, albeit unresolved. Further details can be found in Peimbert (1995), Mathis, Torres-Peimbert, \& Peimbert (1998), and Stasińska (1998). 


\section{Appendix B}

\section{Abundances From Stellar Spectra}

We do not wish to include a complete description of stellar atmospheres in this review as most readers have completed a stellar atmospheres course or have textbooks like Mihalas (1978) on their shelves or have read shorter introductions like chapter 12 of Cowley (1995). However, in the interest of keeping up-to-date, we provide a brief description with emphasis on oft-heard buzzwords. Converting measured absorption feature strengths into abundances requires a model stellar atmosphere that gives the run of physical variables like temperature and pressure with optical depth. With today's fast computers, it is no longer much of a computational burden to compute fairly realistic LTE model atmospheres, most of which have the following features. (1) Plane-parallel geometry. This is a good approximation for most stars, but a spherical geometry is needed for M giants and other stars that have extended envelopes. (2) A modern model atmosphere (e.g. Gustafsson 1989; Kurucz 1993) will be line-blanketed. That is, individual transitions from (usually millions) of atomic and molecular lines are explicitly included in the frequency-dependent opacity calculations along with the various sources of continuous opacity like electron scattering or $\mathrm{H}^{-}$. (3) Convection is often treated in the mixing length approximation, but alternatives are always being tested. (4) For many stars, local thermodynamic equilibrium (LTE) is assumed. In this case the local radiation field and the local thermodynamic state (which can be described by temperature and pressure alone) of the matter are equilibrated. The alternative is non-LTE (NLTE), in which the radiation field decouples from the matter. The complicating part of this is that atomic level occupation numbers then depend mostly on the radiation field rather than the local matter thermodynamics, and the radiation field is not local and therefore a global self-consistent solution must be sought. Extreme NLTE prevails in the case of nebulae and hot stars, while most stars can be treated with the LTE assumption for most lines. However, a temperature inversion above the photosphere (i.e. a chromosphere) will introduce certain NLTE effects in some lines. If these lines are going to be used for abundance analysis, they need to be treated appropriately. See Mihalas (1978) or Kudritzki \& Hummer (1990) for a description of NLTE methods in hot stars.

Except for those stars where spherical geometry effects become important, effective

temperature $\mathrm{T}_{\text {eff }}$, surface gravity $\log \mathrm{g}$ (where $\mathrm{g}$ is expressed in $\mathrm{cm} \mathrm{s}^{-2}$ ), and abundance are sufficient parameters to begin the calculation of a model star. Temperatures can be gotten from calibrations of broad-band or narrow-band colors, by Balmer line strength, or by consideration of two or more ionization states of the same species (by requiring consistent abundance results from all ionization states). Abundance analysis is often not very sensitive to surface gravity. 
There is also the matter of Doppler broadening of lines. Thermal motion of gas particles causes a broadening of the line profile that is almost always larger than the width of the line as broadened by pressure and by radiation damping. In the LTE approximation inclusion of this effect is trivially accomplished by convolving the intrinsic Lorentzian line profile with the Gaussian thermal Doppler profile. But there is also the matter of bulk motions in the atmosphere, in the sun seen as prominences, spicules, granules, acoustical waves, and other phenomena. These add Doppler width to lines, but their velocity distribution is not known. In the face of the total unknown, we follow historical precedent and assume Gaussian random motion, and an empirically adjusted term is added to the width of the Gaussian Doppler profile. That is, inside the Gaussian broadening function $e^{-\left(\Delta \lambda / \Delta \lambda_{\mathrm{D}}\right)^{2}}$,

$$
\frac{\Delta \lambda_{\mathrm{D}}}{\lambda}=\frac{1}{\mathrm{c}} \cdot \frac{2 \mathrm{RT}}{\mu}+\xi_{\mathrm{t}}^{2}
$$

where $\lambda$ is the central wavelength of the line, $\Delta \lambda_{\mathrm{D}}$ is the Doppler width, $\mathrm{R}$ is the gas constant, $\mathrm{T}$ is the temperature, $\mu$ is the mean atomic weight, $\mathrm{c}$ is the light speed, and $\xi_{\mathrm{t}}$ is called the microturbulent velocity, meant to account for Gaussian-random, optically thin turbulent motion in the atmosphere. The final Doppler motion to be considered is macroturbulence, very large moving elements that can be considered independent atmospheres. Such motions will not change the equivalent widths of absorption lines, but they will change the line profile.

Atomic (or molecular) parameters are often crucial to a reliable abundance. Parameters may include damping constants for radiation and van der Waals forces. The damped line profile is usually approximated by a Lorentzian function. The atomic parameters that are mentioned most often are the "gf values", where $g$ is the statistical weight of the level and $\mathrm{f}$ is the oscillator strength; They are usually combined as "log gf." The gf values can be calculated with varying degrees of accuracy, and many can be measured in the laboratory, but the usual method of getting accurate values is to compute a model atmosphere for the sun. The equivalent widths of the line transitions one is interested in studying are measured from the solar atlas, and also computed from the model atmosphere using standard meteoritic + photospheric abundances, for example those of Grevesse, Noels, \& Sauval (1996). The gf values are then adjusted until the model equivalent widths match those of the sun. This method begins to fail for stars too dissimilar from the sun in temperature because the line of interest will be invisible in one of the two stars, or on a different part of the curve-of-growth.

The curve-of-growth is the increase in equivalent width of a line as a function of abundance. When lines are weak, they grow linearly with the abundance. When the line center nears its maximum depth, little growth in equivalent width is seen until the line is so saturated that the weak wings of the line profile contribute. Thus the textbook 
curve-of-growth has a linear-with-abundance portion, an almost flat portion, and then a proportional-to-the-square-root-of-the-abundance portion. For accurate abundance work, it is therefore advantageous to choose weak lines in the linear-growth part of the curve.

Observational material for spectroscopic abundance work will vary according to circumstance. In the ideal case, one would have high $\mathrm{S} / \mathrm{N}$, high resolution $(\mathrm{R}=\lambda / \Delta \lambda=$ 50000 to 100000) spectra compared with near-perfect models. In reality, the models have defects and many/most of the interesting stars are too far away for both high $\mathrm{S} / \mathrm{N}$ and high resolution spectra. The usual solution is to go to lower resolution. The ultimate

low-resolution spectrum is broad-band photometry $(\mathrm{R} \sim 10)$, which can be coaxed to yield good information about $\mathrm{Z}$ with suitable assumptions and calibrations, but little information about individual elements. A resolution at which some elemental abundance information becomes available is about $\mathrm{R} \sim 500$, but only for the strongest, cleanest absorption features. This is the approximate resolution needed for integrated starlight studies.

\section{Appendix C}

\section{Abundances From Integrated Light}

If a galaxy is too distant for individual stars to be spectroscopically analysed one might still hope to learn something from the properties of the integrated starlight summed over all the stars along the line of sight. Broad-band colors or low resolution spectra can be readily obtained for galaxies of sufficient surface brightness. (The surface brightness constraint disqualifies dwarf spheroidals and other low surface brightness galaxies). The question then becomes how to interpret a radial color profile or a radial absorption line strength profile in terms of abundance. This is done by examining the behavior of "simple" stellar populations with age and abundance. A "simple" stellar population is an abstraction of a star cluster, characterized by a single age and a single abundance. One could use real cluster spectra as templates, if one knew the clusters' ages and abundances (e.g. Bica 1988) and if the cluster library brackets the age-metallicity space of interest. The more usual approach is to use a theoretical stellar evolutionary isochrone as the template population. The isochrone gives direct (but theoretical) information on age and abundance.

The first point to realize is that a young stellar population will contain massive stars. O-type stars for the first few tens of millions years, B-type stars for the first few hundred million years, A-type stars until roughly 1 billion years of age. These hot stars make the integrated colors blue, and absorption line strengths weak (except for hydrogen lines, which are strongest in A-type stars). Furthermore, young populations are very bright compared 
to old populations, so they dominate the integrated light if they are present. In the case of spiral galaxies, with their ongoing star formation, getting abundance information from integrated starlight is difficult or impossible because the depth of the absorption features is very strongly modulated by age effects.

Integrated light is useful, therefore, only in "dead" stellar populations where star formation has not occured for some time, so that the OBA-type stars are gone. Most of the light (at optical wavelengths) then comes from FG-type stars at the main sequence turnoff, and KM-type stars on the red giant branch, in roughly equal proportions. Practically speaking, this means that the bulges of spirals and E and S0 galaxies are the targets for integrated light abundance work.

Even in old populations, age effects are the major complication in getting abundance information. One can never be sure that traces of young stars are completely absent, and if they are present they skew the abundance result toward lower values because the metal lines will be weaker. Most isochrone synthesis models (Aaronson et al. 1978; Worthey 1994; Bressan et al. 1994; Vazdekis et al. 1996) show that age effects are muted compared to abundance effects in their impact on the resultant spectrum shape, colors, and line strengths. The null-spectral-change line is $\Delta \log ($ Age $) / \Delta \log \mathrm{Z} \approx-\frac{3}{2}$, so that a factor of 3 age change produces the same spectral change as a factor of 2 change in metallicity. This $-\frac{3}{2}$ slope is approximate, and can be different (but not wildly so) for different colors or spectral indices. In global terms, this is not so bad. For example, if you can be reasonably certain that an object formed in the first half of the universe's history, then your age will be, at most, a factor of $100 \%$ uncertain. This translates to a factor of $\frac{2}{3} \times 100=66 \%$ uncertainty in abundance, if your isochrone model is calibrated correctly. This is 0.2 dex - an impressive accuracy given the large age range allowed. The qualification "if your isochrone model is calibrated correctly" is important, because scatter among different models amounts to $\pm 35 \%$ in age (or roughly $\pm 25 \%$ in $\mathrm{Z}$ via the $\frac{3}{2}$ rule). (Charlot et al. 1996)

Models for integrated light are conceptually simple addition problems, but the ingredients rest on complicated input physics. Stellar evolutionary isochrones are computed from evolutionary tracks of different masses in order to construct a snapshot of the population at a single age. The tracks, in turn, depend on opacities, equations of state, theories of convection and mass loss, and the numerical methods of making a model star. With the addition of an initial mass function the isochrone specifies star number, luminosity, mass, and temperature in stellar bins along the curve. The integrated luminosity at a single wavelength is $\mathrm{L}_{\lambda}=\sum_{\text {isochrone bins }} l_{\lambda \text {,bin }} \mathrm{dN}$, where the number of stars $\mathrm{dN}$ is obtained from an initial mass function $\Phi(\mathrm{M})$ via $\mathrm{dN}=\Phi(\mathrm{M}) \mathrm{dM}$, and where $\mathrm{l}_{\lambda \text {, bin }}$ is the monochromatic 
luminosity of one star in one bin on the isochrone. Perhaps most commonly these days, $l_{\lambda, \text { bin }}$ is obtained from a grid of theoretical model atmosphere fluxes, but empirical libraries are also used.

In dealing with integrated light models, the critical parameter is temperature: for a good model one must make sure that the temperatures along the isochrone are correct at all points, and that the conversion from temperature to color (or flux or line strength) is solid. Relatively small errors in stellar color can propagate almost unattenuated to the integrated colors (Worthey 1994). 


\section{REFERENCES}

Aaronson, M., Cohen, J. G., Mould, J. R., \& Malkan, M. 1978, ApJ, 223, 824

Afflerbach, A., Churchwell, E., \& Werner, M.W. 1997, ApJ, 478, 190

Ajhar, E. A., Blakeslee, J. P., \& Tonry, J. L. 1994, AJ, 108, 2087

Aller, L.H. 1942, ApJ, 95, 52

Aller, L.H. 1984, Physics Of Thermal Gaseous Nebulae (Dordrecht: Reidel)

Arimoto, N., \& Yoshii, Y. 1987, A\&A, 173, 23

Bell, D.J. 1996, PASP, 108, 1139

Bender, R., Burstein, D., \& Faber, S. M. 1993 ApJ, 411, 153

Bender, R., \& Surma, P. 1993, A\&A, 258, 250

Bica, E. 1988, A\&A, 195, 76

Binney, J., \& Merrifield, M. 1998, Galactic Astronomy, (Princeton: Princeton University Press)

Bressan, A., Chiosi, C., Fagotto, F. 1994, ApJS, 94, 63

Butler, K. 1993, I.A.U. Symp. 155, Planetary Nebulae, eds. R Weinberger and A. Acker, p.73

Cameron, L. M. 1985, A\&A, 147, 47

Carlberg, R. G. 1984, ApJ, 286, 416

Carney, B. W., Aguilar, L., Latham, D. W., \& Laird, J. B. 1990, AJ, 99, 201

Carney, B. W., Laird, J. B., Latham, D. W., \& Aguilar, L. 1996, AJ, 112, 668

Carollo, C. M., \& Danziger, I. J. 1994a, MNRAS, 270, 523

Carollo, C. M., \& Danziger, I. J. 1994b, MNRAS, 270, 743

Charlot, S., Worthey, G., \& Bressan, A. 1996, ApJ, 457, 625

Clayton, D. D. 1983, Principles of Stellar Evolution and Nucleosynthesis (University of Chicago Press: Chicago and London) 
Clegg, R.E.S. 1993, I.A.U. Sym. 155, Planetary Nebulae, eds. R Weinberger and A. Acker (Dordrecht: Kluwer) p.549

Cohen, J. G., Blakeslee, J. P., \& Ryzhov, A. 1998, ApJ, 496, 808

Cowley, C. R. 1995, An Introduction of Cosmochemistry (Cambridge: Cambridge University Press)

Cuisinier, F., Acker, A., \& Köppen, J. 1996, A\&A, 307, 215

Davies, R. L., Sadler, E. M., \& Peletier, R. F. 1993, MNRAS, 262, 650

Deslisle, S. 1998, Ph.D. Thesis, Lavál University, Quebec

de Vaucouleurs, G., \& Pense, W.D. 1978, AJ, 83, 1163

Díaz, A.I. 1999, in Chemical Evolution From Zero To High Redshift, ESO Astrophysics Symposia, J.R. Walsh and M.R. Rosa (eds.), Springer, in press.

Durrell, P. R., Harris, W. E., \& Pritchet, C. J. 1994, AJ, 108, 1214

Edmunds, M.G. 1989, in Evolutionary Phenomena in Galaxies, ed. J.E. Beckman \& B.E.J. Pagel (Cambridge: Cambridge Univ. Press), 356

Edmunds, M.G., \& Pagel, B.E.J. 1984, MNRAS, 211, 507

Edmunds, M.G., \& Terlevich, R. 1992, Elements and the Cosmos (Cambridge: Cambridge University Press)

Edvardsson, B., Andersen, J., Gustafsson, B., Lambert, D.L., Nissen, P.E., \& Tomkin, J. 1993, A\&A, 275, 101

Esteban, C., Peimbert, M., Torres-Peimbert, S., \& Escalante, V. 1998, MNRAS, 295, 401

Faber, S. M. 1972, A\&A, 20, 361

Faber, S. M., Wegner, G., Burstein, D., Davies, R. L., Dressler, A., Lynden-Bell, D., \& Terlevich, R. J. 1989, ApJS, 69, 763

Faber, S. M., Worthey, G., \& González, J. J. 1992, in The Stellar Populations of Galaxies, IAU Symp. 149, eds. B. Barbuy \& A. Renzini, (Kluwer: Dordrecht), 255

Ferguson, A.M.N., Gallagher, J.S., \& Wyse, R.F.G. 1998, ApJ, 116, 673

Ferrini, F., Mollá, M., Pardi, M.C., \& Díaz, A.I. 1994, ApJ, 427, 745 
Fesen, R.A., Blair, W.P., \& Kirshner, R.P. 1985, ApJ, 292, 29

Fich, M., \& Silkey, M. 1991, ApJ, 366, 107

Fitzsimmons, A., Dufton, P.L., \& Rolleston, W.R.J. 1992, MNRAS, 259, 489

Fisher, D., Franx, M., \& Illingworth, G. D. 1996, ApJ, 459, 110

Franx, M., \& Illingworth, G. D. 1990, ApJ, 359, L41

Freeman, K. C., 1996, in New Light on Galaxy Evolution, IAU 171, ed. R. Bender \& R. L. Davies (Kluwer, Dordrecht), 3

Friedli, D., Edmunds, M., Robert, C., \& Drissen, L. 1998, Abundance Profiles: Diagnostic Tools For Galaxy History, A.S.P. Conf. Proc., 147 (San Francisco: ASP)

Friel, E. D. \& Janes, K. A. 1993, A\&A, 267, 75

Frogel, J. A., Terndrup, D. M., \& Whitford, A. E. 1990, ApJ, 353, 494

Garnett, D.R. 1998, in Abundance Profiles: Diagnostic Tools For Galaxy History, A.S.P. Conf. Proc., 147, eds: Friedli, D., Edmunds, M., Robert, C., \& Drissen, L. (San Francisco: ASP), p.78

Garnett, D.R. 1989, ApJ, 345, 282

Garnett, D.R., \& Shields, G.A. 1987, ApJ, 317, 82

Garnett, D.R., Shields, G.A., Peimbert, M., Torres-Peimbert, S., Skillman, E.D., Dufour, R.J., Terlevich, E., Terlevich, R.J. 1999, ApJ, in press

Garnett, D.R., Skillman, E.D., Dufour, R.J., Peimbert, M., Torres-Peimbert, S., Terlevich, R.J., Terlevich, E., \& Shields, G.A. 1995, ApJ, 443, 64

Garnett, D.R., Skillman, E.D., Dufour, R.J., \& Shields, G.A. 1997, ApJ, 481, 174

Gehren, T., Nissen, P.E., Kudritzki, R.P., \& Butler, K. 1985, in Proc. ESO Workshop on Production and Distribution of CNO Elements, ed. I.J. Danziger, F. Matteucci, \& K. Kjaer (Garching: European Southern Observatory), 171

Gilmore, G., Wyse, R. F. G., \& Jones, J. B. 1995, AJ, 109, 1095

González, J. J. 1993, Ph. D. thesis, Univ. of California, Santa Cruz, USA 
Grevesse, N., \& Noels, A. 1993, in Origin and Evolution of the Elements, eds. N. Prantzos, E. Vangioni-Flam, \& M. Cassé (Cambridge: Cambridge University Press), 15

Grevesse, N., Noels, A., \& Sauval, A.J. 1996, in ASP Conf. Ser. 99, Cosmic Abundances, ed. S.S. Holt \& G. Sonneborn (San Francisco: ASP), 117

Grillmair, C. J., Lauer, T. R., Worthey, G. Faber, S. M., Freedman, W. L., Madore, B. F., Baum, W. A., Holtzman, J. A., Lynds, C. R., O’Neil, E. J., \& Stetson, P. B. 1996, AJ, 112, 1975

Gummersbach, C.A., Kaufer, A., Schäfer, D.R., Szeifert, T., \& Wolf, B. 1998, A\&A, 338, 881

Gustafsson, B. 1989, ARA\&A, 27, 701

Gustafsson, B., Karlsson, T., Olsson, E., Edvardsson, B., \& Ryde, N. 1999, A\&A, 342, 426

Habing, H.J., \& Lamers, J.G.L.M. 1997, IAU Symp. 180, Planetary Nebulae, (Dordrecht:Kluwer)

Harding, P. 1996, in Formation of the Galactic Bulge, Inside and Out, ASP Conf. Ser. 92, ed. H. Morrison \& A. Sarajedini (Astronomical Society of the Pacific: San Fransisco), 151

Harris, W. E. 1996, AJ, 112, 1487

Henry, R.B.C. 1989, MNRAS, 241, 453

Henry, R.B.C. 1990, ApJ, 356, 229; erratum: ApJ, 363, 728

Henry, R.B.C. 1993, MNRAS, 261, 306

Henry, R.B.C., Balkowski, C., Cayatte, V., Edmunds, M.G., \& Pagel, B.E.J. 1996, MNRAS, 283,635

Henry, R.B.C., \& Howard, J.W. 1995, ApJ, 438, 170

Henry, R.B.C., Kwitter, K.B., \& Dufour, R.J. 1999, ApJ, 517, 000

Hesser, J. E., Stetson, P. B., McClure, R. D., van den Bergh, S., Bolte, M., Harris, W. E., VandenBerg, D. A., Bell, R. A., Fahlman, G. G., Richer, H. B., \& Bond, H. E. 1997, BAAS, 191, 79.01

Howard, J.W., Henry, R.B.C., \& McCartney, S. 1997, MNRAS, 284, 465 
Ibata, R. A., \& Gilmore, G. 1995, MNRAS, 262, 605

Izotov, Y.I., \& Thuan, T.X. 1999, ApJ, 511, 639

Jablonka, P., Martin, P., \& Arimoto, N. 1996, AJ, 112, 1415

Jacoby, G.H., \& Ciardullo, R. 1999, ApJ, in press

Kaler, J.B. 1980, ApJ, 239, 78

Kaufer, A., Szeifert, Th., Krenzin, R., Baschek, B., \& Wolf, B. 1994, A\&A, 289, 740

Kennicutt, R.C., \& Garnett, D.R. 1996, ApJ, 456, 504

Kilian-Montenbruck, J., Gehren, T., Nissen, P. E. 1994, A\&A, 291, 757

Kingsburgh, R.L., \& Barlow, M.J. 1994, MNRAS, 271, 257

Kobulnicky, H.A., \& Skillman, E.D. 1996, ApJ, 471, 211

Kobulnicky, H.A., \& Skillman, E.D. 1998, ApJ, 497, 601

Kochanek, C.S. 1996, ApJ, 457, 228

Köppen, J. 1994, A\&A, 281, 26

Kormendy, J., \& Djorgovski, S. 1989, ARA\&A, 27, 235

Kudritzki, R. P. \& Hummer, D. G. 1990, ARA\&A, 28, 303

Kurucz, R. L. 1993, in Peculiar Versus Normal Phenomena in A-type and Related Stars, ed. M. M. Dworestsky, F. Castellini, \& R. Faraggiana, Proc. IAU Colloqu., 138, 87

Larson, R. B. 1974, MNRAS, 166, 585

Larson, R. B. 1998, MNRAS, 301, 569

Lauroesch, J.T., Truran, J.W., Welty, D.E., \& York, D.G. 1996, PASP, 108, 641

Lee, M. G., Kim, E., \& Geisler, D. 1998, AJ, 115, 947

Lin, D.N.C., \& Pringle, J.E. 1987, ApJ, 320, L87

Liu, X.-W. 1998, MNRAS, 295, 699

Lu, L., Sargent, W.L.W., \& Barlow, T.A. 1996, ApJS, 107, 475 
Maciel, W.J., \& Köppen, J. 1994, A\&A, 282, 436

Maciel, W.J., \& Quireza, C. 1999, A\&A, in press

Maeder, A. 1992, A\&A, 264, 105

Martin, P., \& Roy, J-R. 1994, ApJ, 424, 599

Mathis, J.S., Torres-Peimbert, S., \& Peimbert, M. 1998, ApJ, 495, 328

Matteucci, F., \& Tornambé, A. 1987, A\&A, 185, 51

Mc Call, M.L. 1982, PhD thesis, University of Texas, Austin

Mc Call, M.L., Rybski, P.M., \& Shields, G.A. 1985, ApJS, 57, 1

McCarthy, J.K., Venn, K.A., Lennon, D.J., Kudritzki, R.-P., \& Puls, J. 1998, in BoulderMunich II: Properties of Hot, Luminous Stars, ed.I. Howarth, ASP Conf. Ser. 131 (San Francisco: ASP), 197

McGaugh, S.S. 1991, ApJ, 380, 140

McGaugh, S.S. 1994, ApJ, 426, 135

McWilliam, A. 1997, ARA\&A, 35, 503

Mehlert, D., Bender, R., Saglia, R. P., \& Wegner, G. 1998, in Untangling Coma Berenices: A New Vision of an Old Cluster, eds. A. Mazure, F. Casoli, F. Durret, and D. Gerbal, (Word Scientific Publishing Co Pte Ltd), 107

Mihalas, D. 1978, Stellar Atmospheres, (W. H. Freeman and Company: San Francisco)

Mollá, M., Ferrini, F., \& Diaz, A.I. 1996, ApJ, 466, 668

Monteverde, M.I., Herrero, A., Lennon, D.J., \& Kudritzki, R.-P. 1997, ApJ, 474, 107

Nissen, P. E. \& Schuster, W. J. 1997, A\&A, 326, 751

Nomoto, K., Hashimoto, M., Tsujimoto, T., Thielemann, F.-K., Kishimoto, N., Kubo, Y., \& Nakasato, N. 1997b, Nuc. Phys. A, A616, 79c

Nomoto, K., Iwamoto, K., Nakasato, N., Thielemann, F.-K., Brachwitz, F., Tsujimoto, T., Kubo, Y., \& Kishimoto, N. 1997a, Nuc. Phys. A, A621, 467c

Oey, M.S., \& Kennicutt, R.C. 1993, ApJ, 411, 137 
Osterbrock, D.E. 1989, Astrophysics of Gaseous Nebulae and Active Galactic Nuclei, (University Science Books)

Osterbrock, D.E. 1988, PASP, 100, 412

Ostrov, P. G., Forte, J. C., \& Geisler, D. 1998, AJ, 116, 2854

Pagel, B.E.J., 1997, Nucleosynthesis and Chemical Evolution of Galaxies, (Cambridge: Cambridge University Press)

Pagel, B.E.J., \& Edmunds, M.G. 1981, ARA\&A, 19, 77

Pagel, B.E.J., Edmunds, M.G., Blackwell, D.E., Chun, M.S., \& Smith, G. 1979, MNRAS, 189, 95

Panagia, N., \& Tosi, M. 1981, A\&A, 96, 306

Peimbert, M. 1967, ApJ, 150, 825

Peimbert, M. 1978, Planetary Nebulae, IAU Symp. 76, Y. Terzian, ed. (Dordrecht: Reidel), p. 215

Peimbert, M. 1990, Rep. Prog. Phys., 53, 1559

Peimbert, M. 1995, in The Analysis of Emission Lines, ed. R.E. Williams \& M. Livio (Cambridge: Cambridge Univ. Press), 165

Peimbert, M., Torres-Peimbert, S., \& Dufour, R.J. 1993, ApJ, 418, 760

Peimbert, M., Torres-Peimbert, S., \& Luridiana, V. 1995, Rev. Mex. A. \& Ap. Conf. Ser., 3,296

Peletier, R. F., Davies, R. L., Illingworth, G. D., Davis, L. E., \& Cawson, M. 1990, AJ, 100, 1043

Perinotto, M. 1991, ApJS, 76, 687

Pettini, M., Ellison, S.L., Steidel, C.C., \& Bowen, D.V. 1999, ApJ, 510, 576

Prantzos, N., Vangioni-Flam, E., \& Cassé, M. 1993, Origin and Evolution of the Elements, (Cambridge: Cambridge University Press)

Prantzos, N., Vangioni-Flam, E., \& Chauveau, S. 1994, A\&A, 285, 132

Proctor, R. N., Sansom, A. E., \& Reid, N. 1999, MNRAS, submitted 
Rich, R. M. 1998, in Abundance Profiles: Diagnostic Tools for Galaxy History, ASP Conference Series, ed. D. Friedli, M. Edmunds, C. Robert, \& L. Drissen (San Francisco: ASP), 147, 36

Rich, R. M., Mighell, K. J., \& Neill, J. A. 1996, in Formation of the Galactic Halo: Inside and Out, ASP Conf. Ser. 92, ed. H. L. Morrison \& A. Sarajedini (Astronomical Society of the Pacific: San Francisco), 544

Richer, H. B., Harris, W. E., Fahlman, G. G., et al., 1996, ApJ, 463, 602

Robin, A.C., Haywood, M., Creze, M., Ojha, D.K., \& Bienayme, O. 1996, A\&A, 305, 125

Rolfs, C.E., \& Rodney, W.S. 1988, Cauldrons in the Cosmos (Chicago: University of Chicago Press)

Rose, J. A. 1994, AJ, 107, 206

Rudolph, A.L., Simpson, J.P., Haas, M.R., Erickson, E.F., \& Fich, M. 1997, ApJ, 489, 94

Samland, M. 1998, ApJ, 496, 155

Samland, M., Hensler, G., \& Theis, Ch. 1997, ApJ, 476, 544

Savage, B.D., \& Sembach, K.R. 1996, ARA\&A, 34, 279

Searle, L. 1971, ApJ, 168, 327

Shaver, P.A., McGee, R.X., Newton, L.M., Danks, A.C., Pottasch, S.R. 1983, MNRAS, 204,53

Shields, G.A. 1990, ARA\&A, 28, 525

Shields, G.A., Skillman, E.D., \& Kennicutt, R.C. 1991, ApJ, 371, 82

Shields, J.C., \& Kennicutt, R.C. 1995, ApJ, 454, 807

Simpson, J.P., Colgan, S.W.J., Rubin, R.H., Erickson, E.F., \& Haas, M.R. 1995, ApJ, 444, 721

Skillman, E.D., Kennicutt, R.C. Jr., Shields, G.A., \& Zaritsky, D. 1996, ApJ, 462, 147

Smartt, S.J., \& Rolleston, W. R. J. 1997, ApJ, 481, L47

Snow, T.P., \& Witt, A.N. 1996, ApJ, 468, L65 
Soria, R., Mould, J. R., Watson, A. M., Gallagher, J. S., III, Ballester, G. E., Burrows, C. J., Casertano, S., Clarke, J. T., Crisp, D., Griffiths, R. E., Hester, J. J., Hoessel, J. G., Holtzman, J. A., Scowen, P. A., Stapelfeldt, K. R., Trauger, J. T., \& Westphal, J. A. 1996, ApJ, 465, 79

Spitzer, L. 1978, Physical Processes in the Interstellar Medium (New York: Wiley)

Stasińska, G. 1998, in Abundance Profiles: Diagnostic Tools For Galaxy History, ASP Conf. Ser. 147, ed. D. Friedli, M. Edmunds, C. Robert, \& L. Drissen (San Francisco: ASP), 142

Stasińska, G., Richer, M.G., \& Mc Call, M.L. 1998, A\&A, 336, 667

Stasińska, G., \& Schaerer, D. 1997, A\&A, 322, 615

Terndrup, D. M. 1988, AJ, 96, 894

Terndrup, D. M. Frogel, J. A., \& Whitford, A. E. 1990, ApJ, 357, 453

Thogersen, E. N., Friel, E. D., \& Fallon, B. V. 1993, PASP, 105, 1253

Thurston, T.R., Edmunds, M.G., \& Henry, R.B.C. 1996, MNRAS, 283, 990

Tinsley, B.M 1980, Fund. Cos. Phys., 5, 287

Trager, S. C., Worthey, G., Faber, S. M., Burstein, D., \& González, J. J. 1998, ApJS, 116, 1

Trimble, V. 1991, Astron. Ap. Rev., 3, 1

Twarog, B. A., Ashman, K. A., \& Anthony-Twarog, B. J. 1997, AJ, 114, 2556

van den Bergh, S. 1962, AJ, 67, 486

van den Bergh, S. 1995, AJ, 110, 2700

van den Hoek, L.B., \& Groenewegen, M.A.T. 1997, A\&AS, 123, 305

van Zee, L., Salzer, J.J., Haynes, M.P., O'Donoghue, A.A., Balonek, T.J. 1998, AJ, 116, 2805.

Vazdekis, A., Casuso, E., Peletier, R. F., Beckman, J. E., 1996, ApJS, 106, 307

Venn, K. A. 1995, ApJS, 99, 659

Venn, K. A. 1998, ApJ, in preparation 
Vila-Costas, M.B., \& Edmunds, M.G. 1992, MNRAS, 259, 121

Vila-Costas, M.B., \& Edmunds, M.G. 1993, MNRAS, 265, 199

Vílchez, J.M., \& Esteban, C. 1996, MNRAS, 280, 720

Walsh, J.R., \& Rosa, M.R. 1999, Chemical Evolution From Zero To High Redshift, ESO Astrophysics Symposia (Springer), in press

Weiss, A., Peletier, R. F., \& Matteucci, F. 1995, A\&A, 296, 73

Wheeler, J.C., Sneden, C., \& Truran, J.W. 1989, ARA\&A, 27, 279

White, S. D. M. 1980, MNRAS, 191, 1P

Wielen, R., Fuchs, B., \& Dettbarn, C. 1996, A\&A, 314, 438

Williams, R.E., \& Livio, M. 1995, The Analysis of Emission Lines, (Cambridge: Cambridge University Press)

Woosley, S.E., \& Weaver, T.A. 1995, ApJS, 101, 181

Worthey, G. 1998, PASP, 110, 888

Worthey, G. 1994, ApJS, 95, 107

Worthey, G., Dorman, B., \& Jones, L. A. 1996, AJ, 112, 948

Worthey, G., Faber, S. M., González, J. J., \& Burstein, D. 1994, ApJS, 94, 687

Worthey, G., Faber, S. M., González, J. J. 1992, ApJ, 398, 69

Worthey, G., \& Ottaviani, D. L. 1997, ApJS, 111, 377

Wyse, R. F. G., \& Gilmore G. 1995, AJ, 110, 2771

Yoshii, Y., \& Sommer-Larsen, J. 1989, MNRAS, 236, 779

Zaritsky, D. 1992, ApJ, 390, L73

Zaritsky, D., Kennicutt, R.C., \& Huchra, J.P. 1994, ApJ, 420, 87

Zepf, S. E., \& Ashman, K. M. 1993, MNRAS, 264, 611

Zinn, R. 1996, in Formation of the Galactic Halo, Inside and Out, ASP Conf. Ser., 92, ed. H. Morrison \& A. Sarajedini, (Astronomical Society of the Pacific, San Francisco), 211 
Fig. 1. - $12+\log (\mathrm{O} / \mathrm{H})$ vs. galactocentric distance $(\mathrm{kpc})$ for the several emission-line studies of the Milky Way. First author of each study is given in each panel, where references are given in Table 2 . Solid lines are least square fits to the points, while dot-dashed lines show the composite fit for comparison. Parameters for the fits are given in Table 2. Typical uncertainties for all the data sets are shown with error bars in the bottom-left corner. Note that Vílchez \& Esteban (1996) employed two methods for obtaining abundances. See their paper for an explanation.

Fig. 2.- $12+\log (\mathrm{O} / \mathrm{H})$ versus galactocentric distance in $\mathrm{kpc}$ for the data sets of Afflerbach et al. (1997, A); Fesen et al. (1985; F); Fich \& Silkey (1991; C); Maciel \& Köppen (1994, M); Rudolph et al. (1996, R); Shaver et al. (1983, S); and Vílchez \& Esteban (1996, V). Filled and open circles are for B star data from Smartt \& Rolleston (1997) and Gummersbach et al. (1998), respectively. Typical uncertainties for all the data sets are indicated in the bottom-left corner. Lines represent models by Pagel (1997; dashed line), Samland et al. (1997; solid line), Ferrini et al. (1994; dot-dashed line), and Köppen (1994 and private communication; long-dashed line). The sun's position is indicated with an ' $\mathrm{x}$ '.

Fig. 3. - $12+\log (\mathrm{O} / \mathrm{H})$ versus galactocentric distance in isophotal radius for the Milky Way (filled circles), M33 (open circles), and NGC 628 (open squares). Data sources are given in the text, while gradient slopes in $\operatorname{dex} / R_{o}$ are given in the legend.

Fig. 4a.- Characteristic abundances (top three panels) and gradient slopes (bottom three panels) from Zaritsky et al. (1994) plotted against morphological T Type, absolute blue magnitude $\mathrm{M}_{\mathrm{B}}$, and circular velocity, $\mathrm{V}_{\mathrm{c}}$ in $\mathrm{km} / \mathrm{s}$. Regular and barred spirals are shown with filled and open circles, respectively. Milky Way data (see text for references) are shown with plusses.

Fig. 4b.- Oxygen abundance at one effective radius expressed as $12+\log (\mathrm{O} / \mathrm{H})$ versus log galaxy mass in solar units for a small sample of spirals. Abundance data are from Garnett \& Shields (1987), Skillman et al. (1996), and Henry et al. (1996). Sources for galaxy masses are given in Henry et al. The two points connected by a line are for NGC 753, whose mass was determined for $\mathrm{H}_{\mathrm{o}}$ values of 50 and $100 \mathrm{~km} / \mathrm{s}$.

Fig. 5. - $12+\log (\mathrm{O} / \mathrm{H})$ of extragalactic $\mathrm{H}$ II regions versus the logarithm of the total surface density $\Sigma\left(\mathrm{M}_{\odot} / \mathrm{pc}^{2}\right)$ at the corresponding position, from Vila-Costas \& Edmunds (1992). Filled and open squares represent objects from early (Sab-Sc) and late (Scd-Irr) spirals, respectively. Copyright Blackwell Science Ltd., reproduced with permission. 
Fig. 6. - $\log (\mathrm{N} / \mathrm{O})$ versus $12+\log (\mathrm{O} / \mathrm{H})$ for $\mathrm{H}$ II regions in the Milky Way disk, extragalactic spirals, and irregulars. Data for the Milky Way are from Afflerbach et al. (1997, A); Fich \& Silkey (1991, F); Shaver et al. (1983, S); Rudolph et al. (1997, R); and Vílchez \& Esteban (1996, V). Extragalactic data are from Izotov \& Thuan (1999, i); Kobulnicky \& Skillman (1996, K); Thurston et al. (1996, T); and van Zee et al. (1998, Z). The circle indicates the position of the Orion Nebula (Esteban et al. 1998), the large S shows the position of the sun (Grevesse et al. 1996), and the L symbols at extremely low oxygen show upper limits for two high redshift damped Lyman- $\alpha$ objects in Lu et al. (1996). Model tracks are calculated from information in VilaCostas \& Edmunds (1993) and are indicated with solid curves. Typical uncertainties are shown in the upper left.

Fig. 7. - $\log (\mathrm{C} / \mathrm{O})$ versus $12+\log (\mathrm{O} / \mathrm{H})$ for extragalactic H II regions. Data are from Garnett et al. (1995, 1997, 1998, G) and Kobulnicky \& Skillman (1998, K), while M and $\mathrm{S}$ show the positions of the Galactic H II region M8 and the sun, respectively. The filled circles show F and G stellar data from Gustafsson et al. (1999). Garnett et al. employed two different reddening laws (see text), and hence these points are joined by vertical lines. Typical uncertainties are shown in the upper left. Regression lines are explained in the text.

Fig. 8.- Logarithmic Ne/O, S/O, and $\mathrm{Ar} / \mathrm{O}$ vs $12+\log (\mathrm{O} / \mathrm{H})$ taken from information in numerous Galactic and extragalactic studies of $\mathrm{H}$ II regions and planetary nebulae. Symbols are defined in the captions of Figs. 2, 6, and 7. The horizontal lines show stellar yield predictions from Nomoto et al. (1997a; dashed lines), Woosley \& Weaver (1995; dot-dashed lines), and Samland (1998; solid lines) for massive star yields integrated over a Salpeter initial mass function and corrected to give ratios by number. Typical uncertainties are indicated with error bars.

Fig. 9.- Abundances of various tracers are plotted as a function of Galactocentric radius. If nothing else, the complexity of the chemical history of the Milky Way should be evident from this figure. The gross trend with radius and age is pronounced. Abundances of globular clusters were taken from Harris (1996; the May 1997 electronic version) and average $\mathrm{M}$ giant metallicities at $\mathrm{b}=-3^{\circ}$ in Baade's window and at $\mathrm{b}=-12^{\circ}$ were taken from Terndrup et al. (1990). FG dwarf data comes from Edvardsson et al. (1993), who were able to separate their stars into age bins. We have averaged over their tabulated age bins to produce "old" and "young" average gradients. As in figure 2, the B star data of Smartt et al. (1997) are shown along with nebular data from Vílchez \& Esteban (1996; "V"), Shaver et al. (1983; "S"), and Afflerbach et al. (1997; "A"). 
Fig. 10.- Mid-optical spectra of stars of differing spectral types are shown, with pseudocontinuum and central index passbands from the Lick index definitions shown. The hashed regions are the central passbands. Solid horizontal line segments mark the pseudocontinua on selected spectra. Dashed lines represent the "continuum" calculated by finding the average flux in the pseudocontinuum passbands and then drawing a straight line between pseudocontinuum midpoints. Indices are expressed either as equivalent widths as in stellar spectroscopy, or as flux ratios in magnitudes, depending on the index. See Worthey et al. (1994) for the details.

Fig. 11.- A. Gradients measured in early-type galaxies in two data sets: González (1993) nearby ellipticals and Mehlert et al. (1998) ellipticals and S0s in the rich Coma cluster. Gradients were derived using the $\mathrm{H} \beta$ index versus a mean abundance index called $[\mathrm{MgFe}]$, defined as $(\mathrm{Mg} \mathrm{b} \times<\mathrm{Fe}>)^{1 / 2}$, where $<\mathrm{Fe}>$ is the arithmetic average of Fe5270 and Fe5335. Derived from this particular index combination and filtered through Worthey (1994) models, the mean age gradient is 0.1 dex/decade (younger toward the center) and the mean gradient in abundance is -0.25 dex/decade (more metal-rich toward the center). Interestingly, the Mehlert gradients in both age and Z are a factor of two more shallow than the González gradients, but as of this writing it is not clear if this is an aperture effect due to the greater distance of the Coma galaxies, or a real environmental trend. B. The correlation between age gradients and $\mathrm{Z}$ gradients. The correlation may have an astrophysical component, but errors from $\mathrm{H} \beta$ emission corrections, abundance ratio effects, and observational error probably explain most of the elongation, which is close to the $-3 / 2$ degeneracy direction expected from a random error source.

Fig. 12. - Models and galaxies are shown in the $<\mathrm{Fe}>$ versus $\mathrm{Mg}_{2}$ diagram. $<\mathrm{Fe}>$ is the arithmetic average of Fe5270 and Fe5335 indices. Each dotted line connects single-age models of $[\mathrm{Z} / \mathrm{H}]=-0.25,0.0$, and 0.25 dex, lower left to upper right in order. The three lines are (from weak to strong $\mathrm{Mg}_{2}$ index strength) for ages 2, 9, and 18 Gyr. Composite combinations of age and metallicity add approximately as vectors. The models are the same in the three panels. Spiral nucleus/bulge data appear in the top panel, S0 galaxy data in the middle panel, and elliptical galaxy data in the bottom. Solid lines trailing from the nuclear data points are trends with galaxy radius in the studies that include radial gradient information. The Galactic bulge (BW = "Baade's Window") is included in the top panel. The tendency of most elliptical nuclei to lie to the right (to higher $\mathrm{Mg}_{2}$ strength) of the model lines is interpreted as a real overabundance of $\mathrm{Mg}$ compared to Fe. Spiral bulges show almost no $[\mathrm{Mg} / \mathrm{Fe}]$ enhancement, but Es show a substantial amount, with S0 galaxies intermediate. Gradients tend to align with the model age-metallicity direction rather 
than the relation linking nuclei, suggesting that $[\mathrm{Mg} / \mathrm{Fe}]$ is more than a nucleusonly phenomenon. The references are: Proctor et al. (1999), Jablonka et al. (1996), Vazdekis et al. (1996), Deslisle (1998), Carollo \& Danziger (1994a and b), "FFI" is Fisher et al. (1996), Davies et al. (1993), and "Lick" is Trager et al. (1998).

Fig. 13. - The $<\mathrm{Mg}_{2}>-\sigma$ relation, data from Faber et al, (1989) and Bender et al. (1993). A regression line of $\left\langle\mathrm{Mg}_{2}>=-0.166+0.20 \log \sigma\right.$ determined by Bender et al. (1993) is shown. Note that the $<\mathrm{Mg}_{2}>$ index differs from the standard definition (found in Worthey et al. 1994) of $\mathrm{Mg}_{2}$. See Trager et al. (1998) to convert between the two systems. This relatively tight scaling relation is useful in galaxy evolution studies because younger-aged galaxies drift toward weaker $\mathrm{Mg}_{2}$ at constant velocity dispersion.

Fig. 14.- Lick index models and $\mathrm{E}+\mathrm{S} 0$ galaxy nuclei are shown with model lines of ages 9 and $18 \mathrm{Gyr}$, connecting $[\mathrm{Z} / \mathrm{H}]=-0.5,-0.25,0.0,0.25$, and 0.5 dex. In the cases of $\mathrm{CN}_{1}, \mathrm{Mg}_{2}$, and $\mathrm{Na} \mathrm{D}$, large elliptical galaxies (solid symbols) lie significantly off the model sequence, indicating light element enhancement. Ca4455, on the other hand, tracks Fe in a scaled-solar fashion (Worthey 1998).

This preprint was prepared with the AAS IATEX macros v4.0. 
Table 1. Milky Way Nebular Studies

\begin{tabular}{lcccccccc}
\hline \hline \multicolumn{1}{c}{ Object } & Author $^{1}$ & No. & Range $(\mathrm{kpc})$ & $\frac{\mathrm{O}}{\mathrm{H}}$ & $\frac{\mathrm{N}}{\mathrm{O}}$ & $\frac{\mathrm{Ne}}{\mathrm{O}}$ & $\frac{\mathrm{S}}{\mathrm{O}}$ & $\frac{\mathrm{Ar}}{\mathrm{O}}$ \\
\hline H II (OPT,RAD) & Shaver & 21 & $5.9-13.7$ & 21 & 20 & 9 & 7 & 16 \\
H II (FIR) & Simpson & 22 & $0.0-10.2$ & 22 & 22 & 17 & 22 & $\cdots$ \\
H II (FIR) & Afflerbach & 34 & $0.0-11.4$ & 34 & 34 & $\cdots$ & 34 & $\cdots$ \\
H II (OPT) & Vílchez & 18 & $11.7-18.0$ & 9 & 9 & $\cdots$ & 6 & $\cdots$ \\
H II (OPT) & Fich & 18 & $11.5-17.9$ & 4 & 4 & $\cdots$ & $\ldots$ & 1 \\
H II (FIR) & Rudolph & 5 & $12.9-17.0$ & 5 & 2 & $\ldots$ & 4 & $\cdots$ \\
PNe II (OPT) & Maciel & 91 & $4.6-12.4$ & 91 & $\ldots$ & 76 & 77 & 73 \\
SNR (OPT) & Fesen & 13 & $4.6-13.5$ & 13 & 13 & $\cdots$ & 13 & $\cdots$ \\
\hline
\end{tabular}

${ }^{1}$ Shaver: Shaver et al. (1983); Simpson: Simpson et al. (1995); Afflerbach et al. (1997); Vílchez: Vílchez \& Esteban (1996); Fich: Fich \& Silkey (1991); Rudolph: Rudolph et al. (1997); Maciel: Maciel \& Köppen (1994); Fesen: Fesen, Blair, \& Kirshner (1985) 
Table 2. Milky Way Oxygen Gradients

\begin{tabular}{lccc}
\hline \hline \multicolumn{1}{c}{ Author } & $\mathrm{G}(\mathrm{dex} / \mathrm{kpc})$ & $\mathrm{A}_{8.5}$ & $\mathrm{c}$ \\
\hline Shaver & $-0.05 \pm 0.01$ & $8.77 \pm 0.14$ & -0.69 \\
Simpson & $-0.06 \pm 0.02$ & $8.57 \pm 0.15$ & -0.56 \\
Afflerbach & $-0.06 \pm 0.01$ & $8.61 \pm 0.09$ & -0.66 \\
Maciel & $-0.07 \pm 0.01$ & $8.66 \pm 0.06$ & -0.70 \\
Fesen & $-0.04 \pm 0.03$ & $8.63 \pm 0.32$ & -0.31 \\
Composite & $-0.06 \pm 0.01$ & $8.68 \pm 0.05$ & -0.63 \\
\hline
\end{tabular}


Table 3. Heavy Element Abundance Ratio Averages ${ }^{1}$

\begin{tabular}{lccc}
\hline \hline \multicolumn{1}{c}{ Sample $^{2}$} & $\log \mathrm{Ne} / \mathrm{O}$ & $\log \mathrm{S} / \mathrm{O}$ & $\log \mathrm{Ar} / \mathrm{O}$ \\
\hline Shaver $(9,7,16)$ & $-0.69 \pm 0.16$ & $-1.40 \pm 0.19$ & $-2.29 \pm 0.16$ \\
Maciel $(76,77,73)$ & $-0.66 \pm 0.12$ & $-1.70 \pm 0.22$ & $-2.27 \pm 0.14$ \\
Garnett $(0,36,0)$ & $\ldots$ & $-1.62 \pm 0.14$ & $\ldots$ \\
van Zee $(56,173,129)$ & $-0.62 \pm 0.18$ & $-1.48 \pm 0.26$ & $-2.24 \pm 0.17$ \\
Izotov $(54,49,53)$ & $-0.72 \pm 0.05$ & $-1.55 \pm 0.06$ & $-2.25 \pm 0.09$ \\
Total $(195,342,271)$ & $-0.67 \pm 0.14$ & $-1.55 \pm 0.24$ & $-2.25 \pm 0.15$ \\
Solar $^{3}$ & -0.79 & -1.54 & -2.35 \\
Orion $^{4}$ & -0.75 & -1.46 & -1.84 \\
Helix $^{5}$ & -0.48 & -2.49 & -2.17 \\
\hline
\end{tabular}

${ }^{1}$ Arithmetic averages, i.e. log mean antilog

${ }^{2}$ Last name of first author of published sample. Numbers in parentheses indicate sample size for $\mathrm{Ne} / \mathrm{O}, \mathrm{S} / \mathrm{O}$, and $\mathrm{Ar} / \mathrm{O}$, respectively

${ }^{3}$ Grevesse et al. 1996

${ }^{4}$ Esteban et al. 1998

${ }^{5}$ Henry, Kwitter, \& Dufour 1999 
Table A1. Prominent Emission Lines In Nebulae

\begin{tabular}{cccccc}
\hline \hline Ion & Wavelength $(\AA)$ & Excitation $^{1}$ & Ion & Wavelength $(\AA)$ & Excitation \\
\hline $\mathrm{C} \mathrm{IV}$ & 1549 & $\mathrm{C}$ & $\mathrm{H} \mathrm{I}$ & 4861 & $\mathrm{R}$ \\
$\mathrm{He} \mathrm{II}$ & 1640 & $\mathrm{R}$ & {$[\mathrm{O} \mathrm{III}]$} & 4959,5007 & $\mathrm{C}$ \\
$\mathrm{C} \mathrm{III}]$ & 1909 & $\mathrm{C}$ & {$[\mathrm{N} \mathrm{II}]$} & 5199 & $\mathrm{C}$ \\
{$[\mathrm{O} \mathrm{II}]$} & 3727 & $\mathrm{C}$ & $\mathrm{He} \mathrm{I}$ & 5876 & $\mathrm{R}$ \\
{$[\mathrm{Ne} \mathrm{III}]$} & 3869,3968 & $\mathrm{C}$ & {$[\mathrm{O} \mathrm{I}]$} & 6300,6360 & $\mathrm{C}$ \\
$\mathrm{He} \mathrm{I}$ & 3889 & $\mathrm{R}$ & {$[\mathrm{S} \mathrm{III}]$} & 6312 & $\mathrm{C}$ \\
{$[\mathrm{S} \mathrm{II}]$} & 4072 & $\mathrm{C}$ & {$[\mathrm{N} \mathrm{II}]$} & 6548,6584 & $\mathrm{C}$ \\
$\mathrm{H} \mathrm{I}$ & 4101 & $\mathrm{R}$ & $\mathrm{H} \mathrm{I}$ & 6563 & $\mathrm{R}$ \\
$\mathrm{H} \mathrm{I}$ & 4340 & $\mathrm{R}$ & {$[\mathrm{S} \mathrm{II}]$} & 6716,6731 & $\mathrm{C}$ \\
{$[\mathrm{O} \mathrm{III}]$} & 4363 & $\mathrm{C}$ & {$[\mathrm{Ar} \mathrm{III}]$} & 7135 & $\mathrm{C}$ \\
$\mathrm{He} \mathrm{I}$ & 4471 & $\mathrm{R}$ & {$[\mathrm{O} \mathrm{II}]$} & 7325 & $\mathrm{C}$ \\
$\mathrm{He}$ II & 4686 & $\mathrm{R}$ & {$[\mathrm{S} \mathrm{III}]$} & 9069,9532 & $\mathrm{C}$ \\
\hline
\end{tabular}

${ }^{1}$ Excitation mechanism, where $\mathrm{C}=$ collisional, $\mathrm{R}=$ recombination 

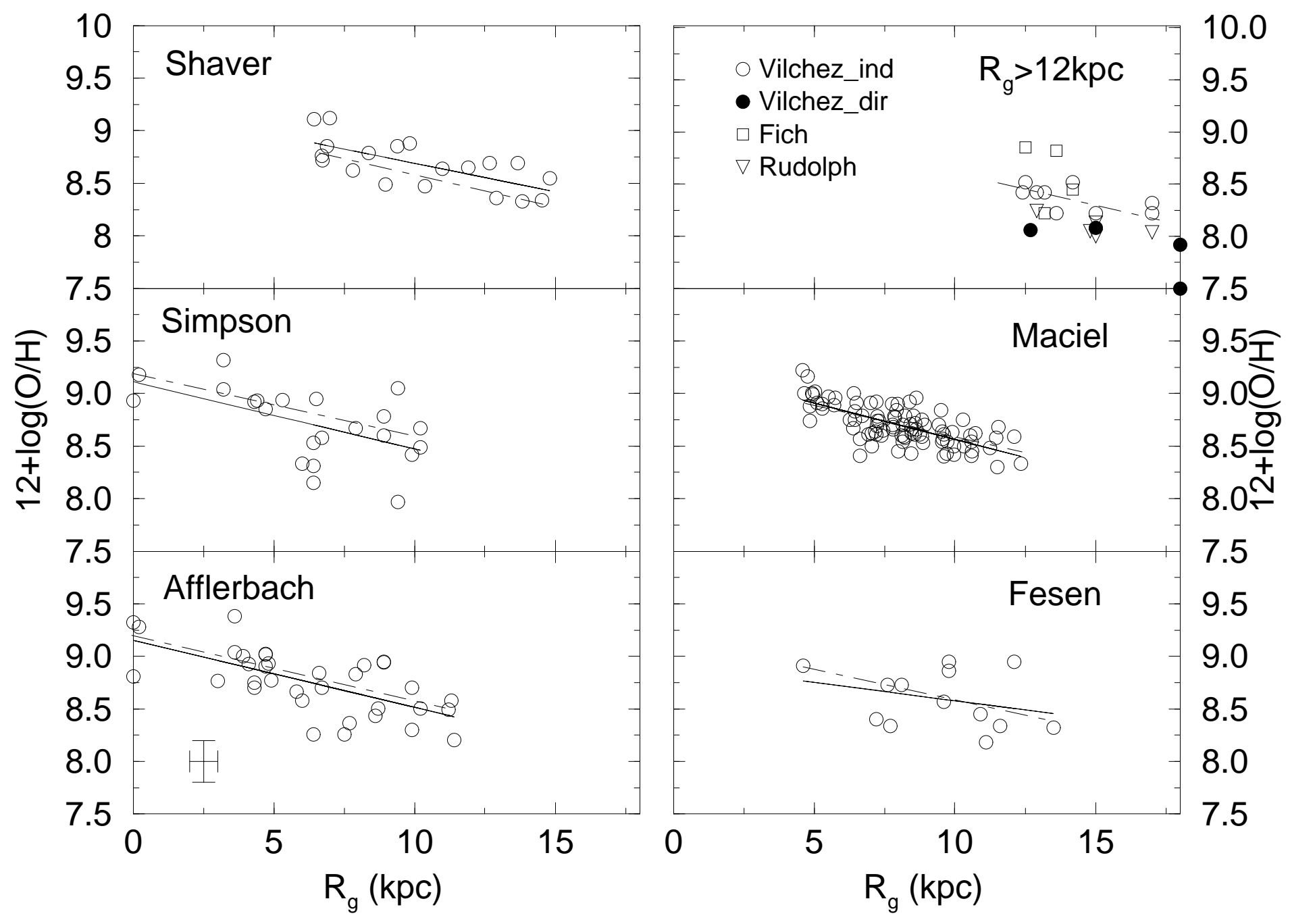


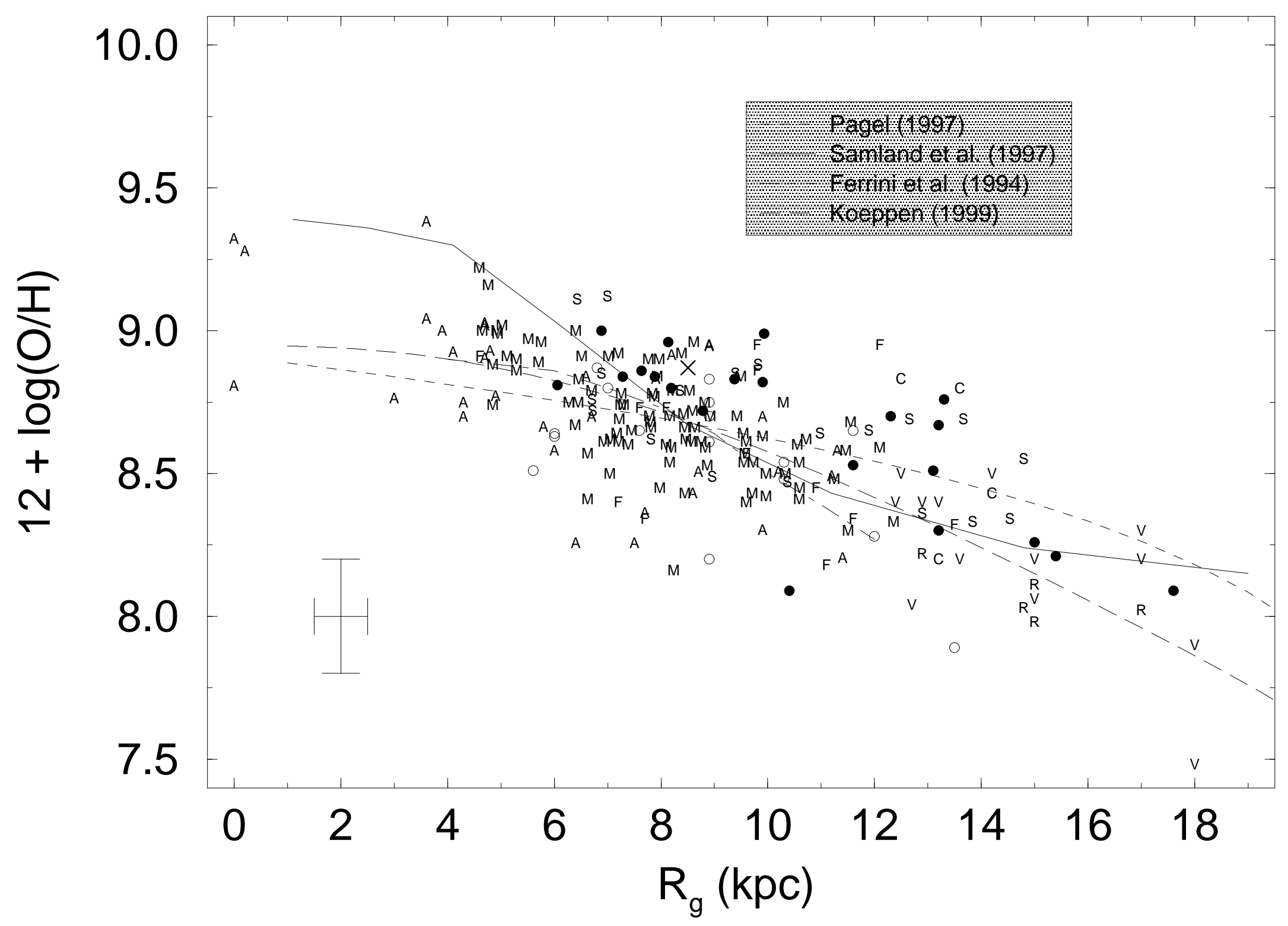




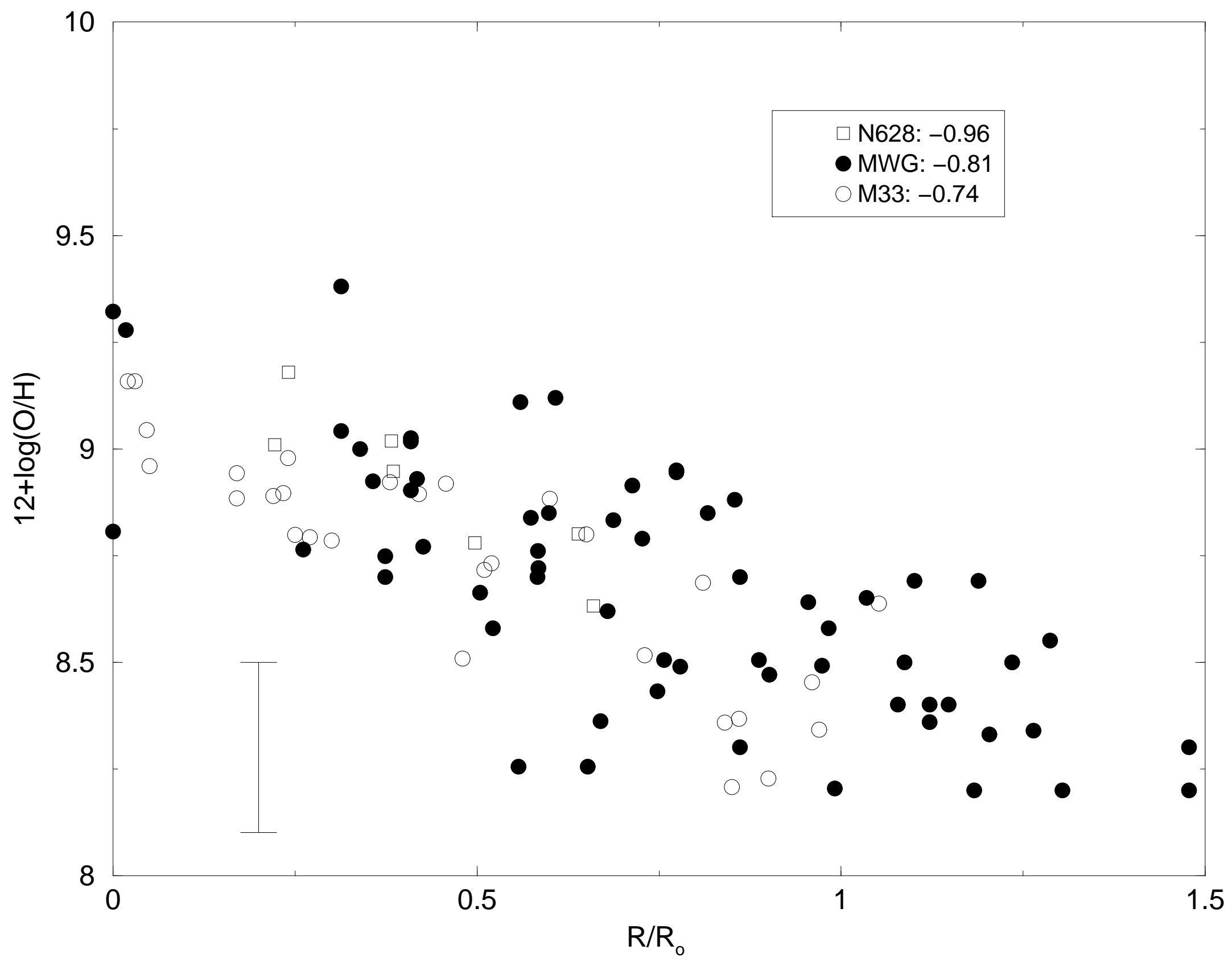




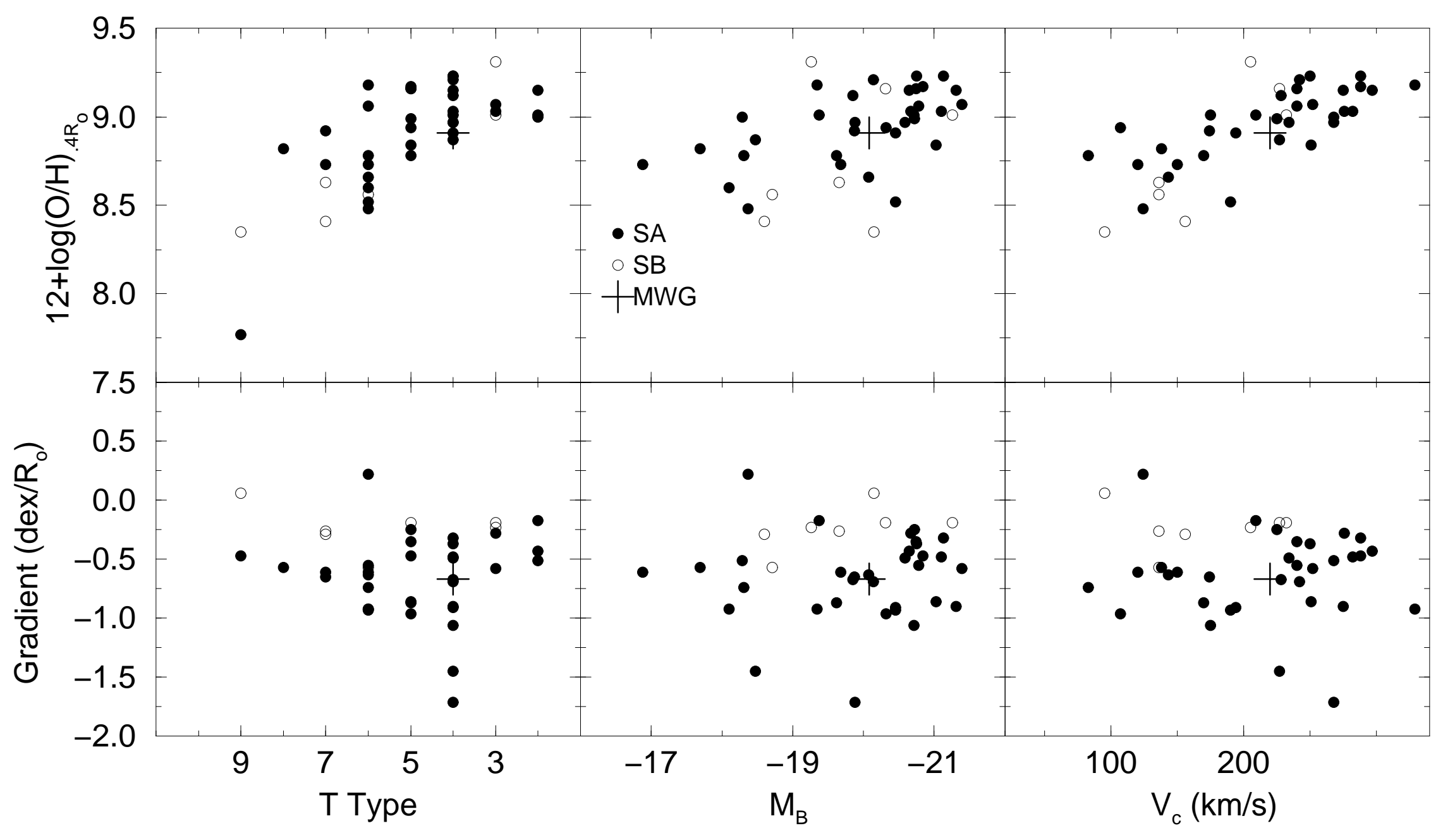




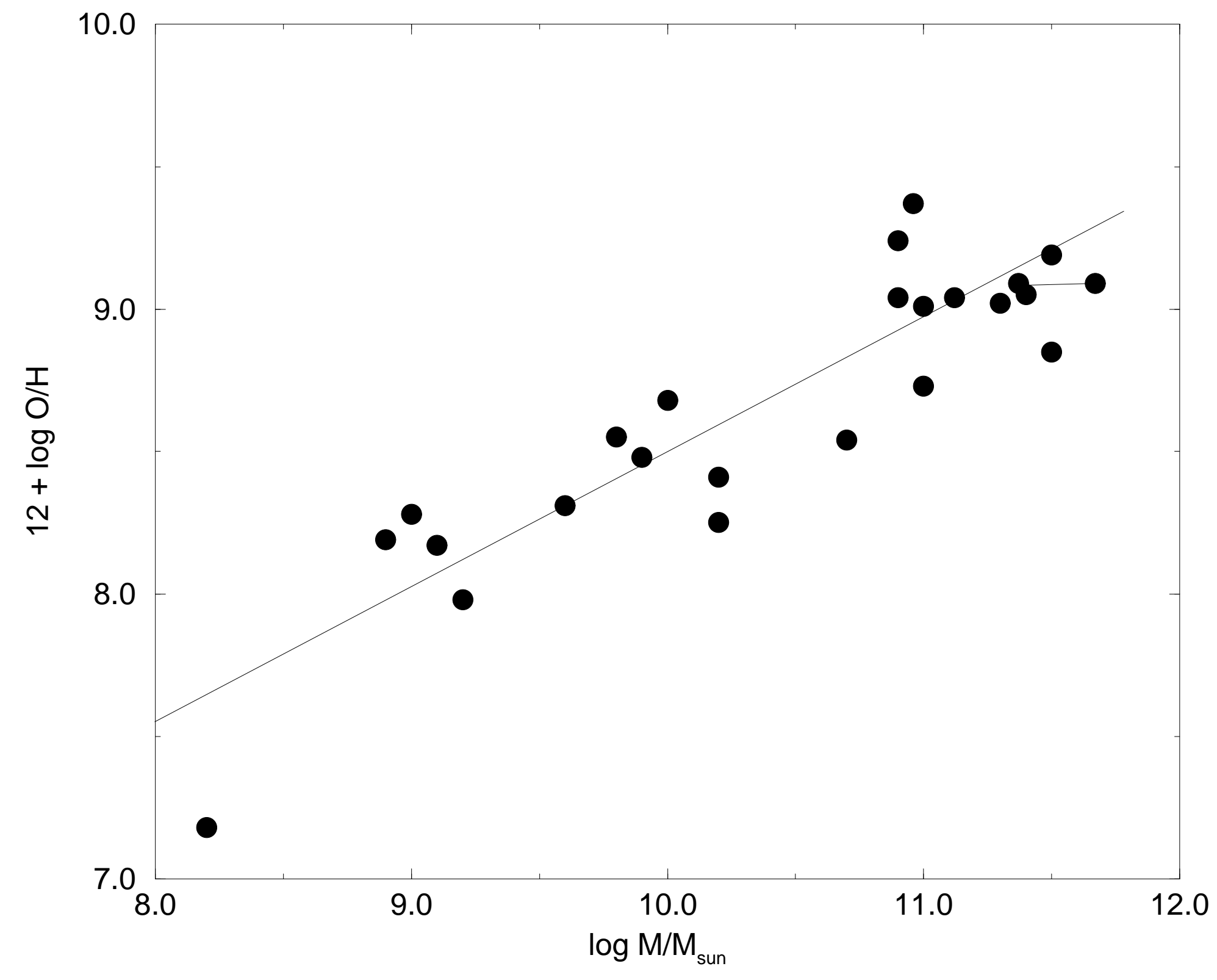




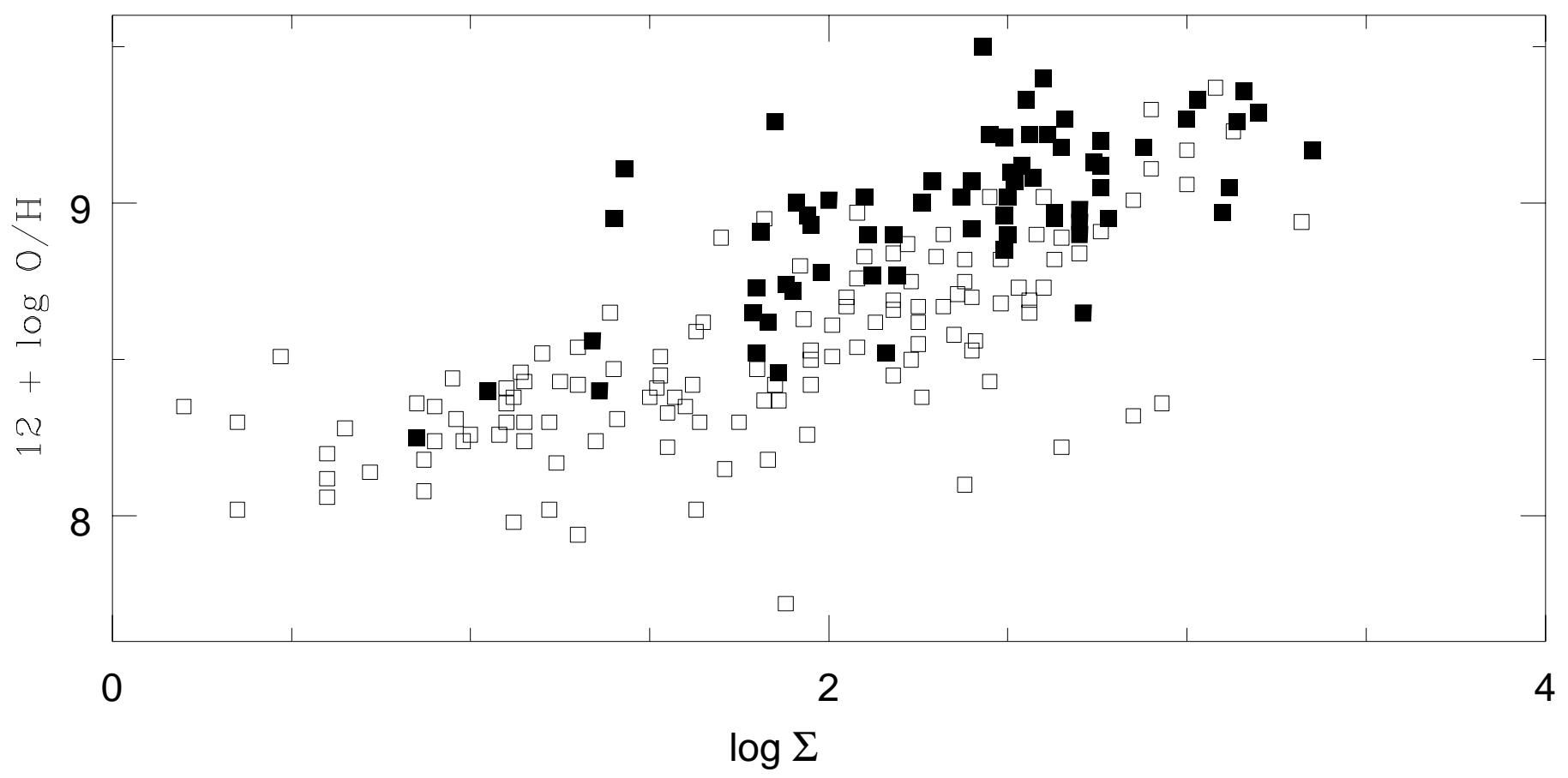




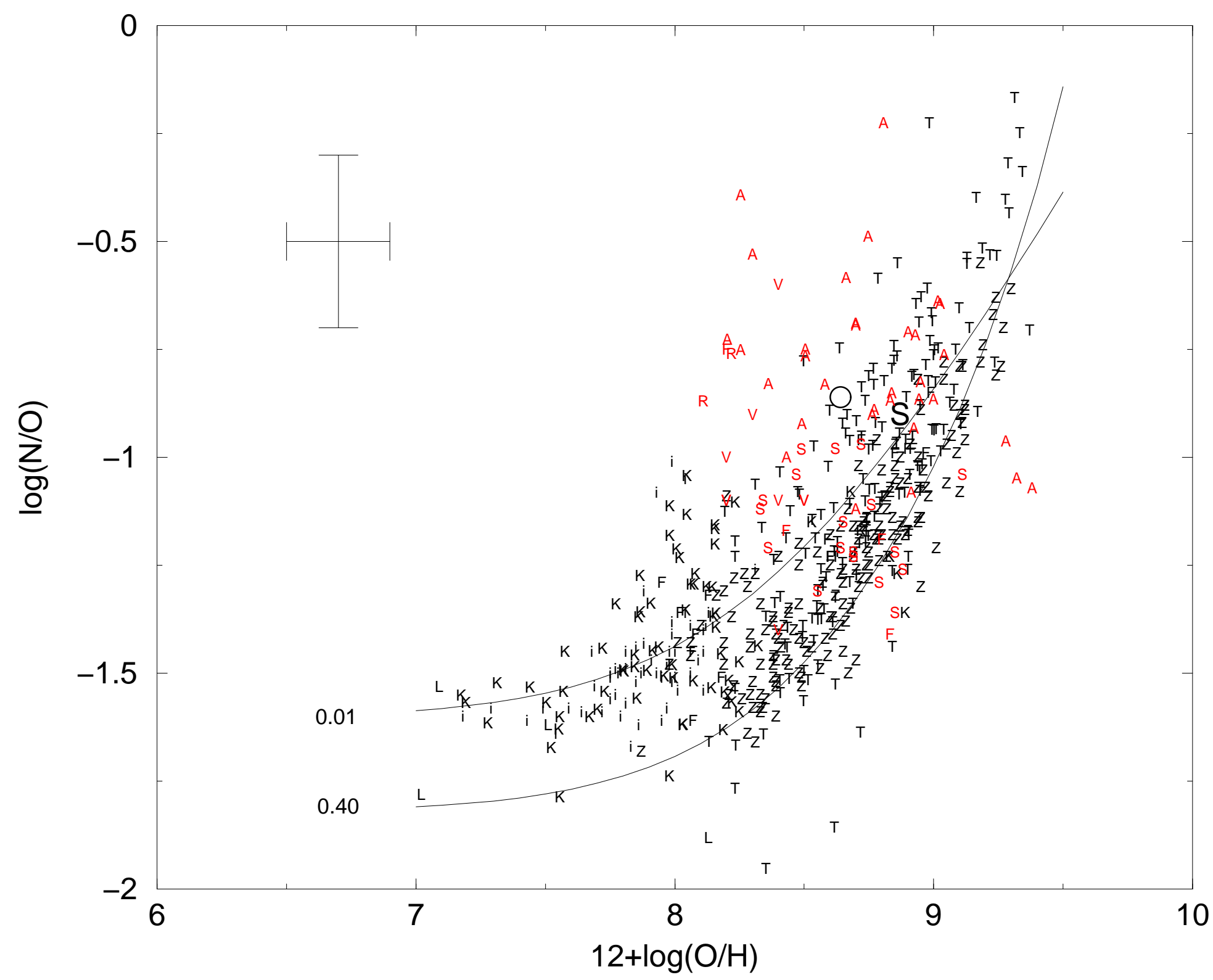




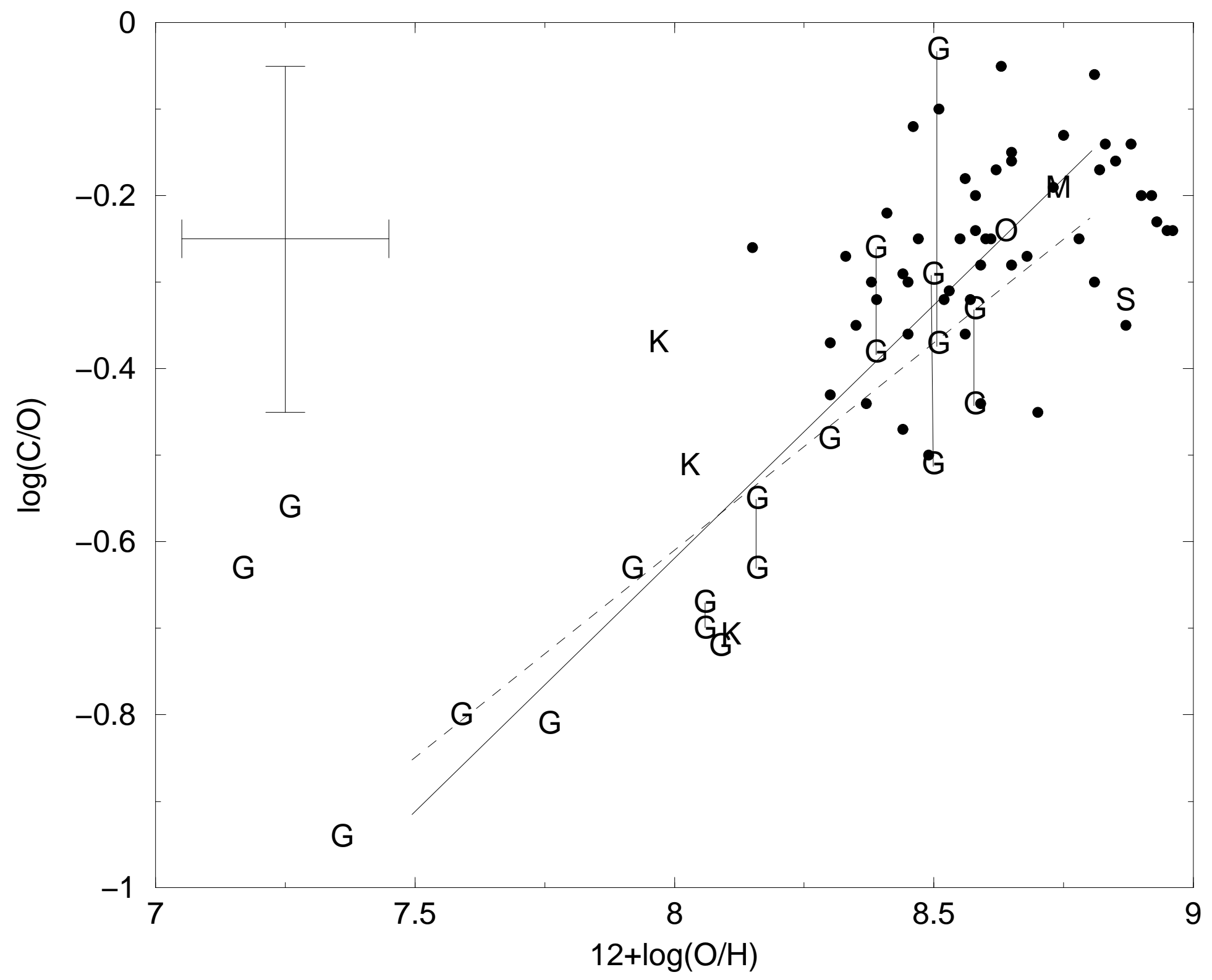




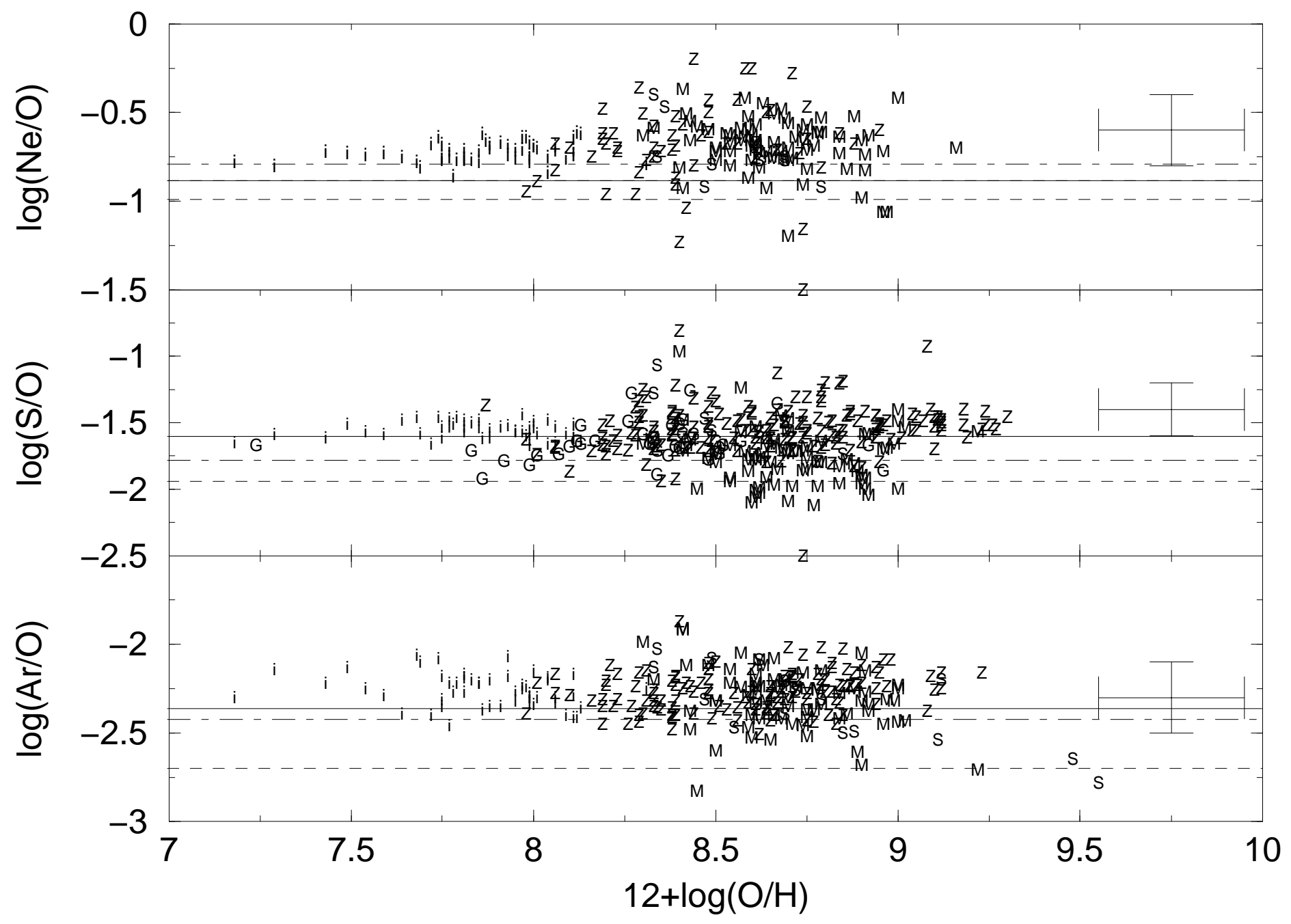




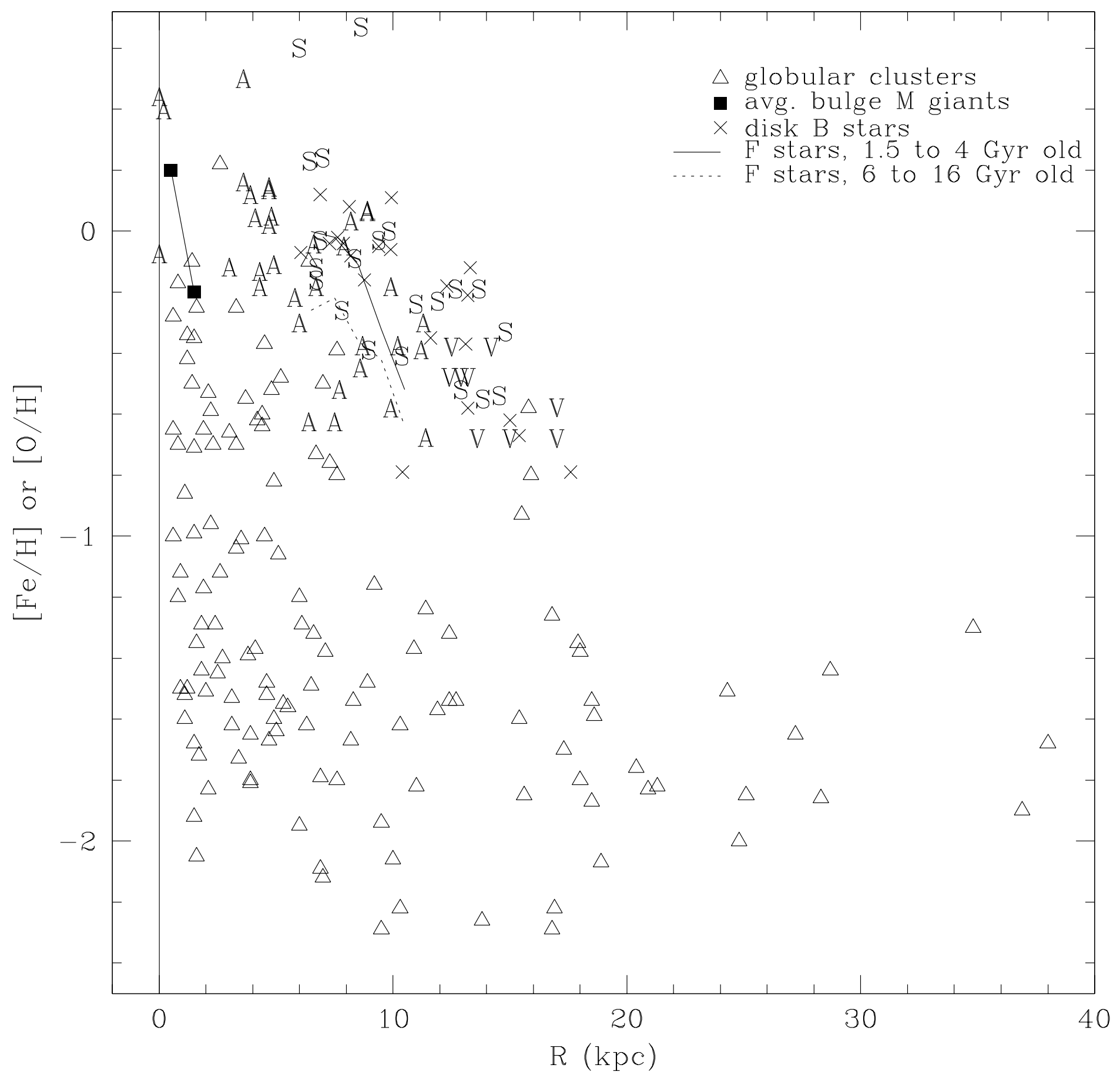




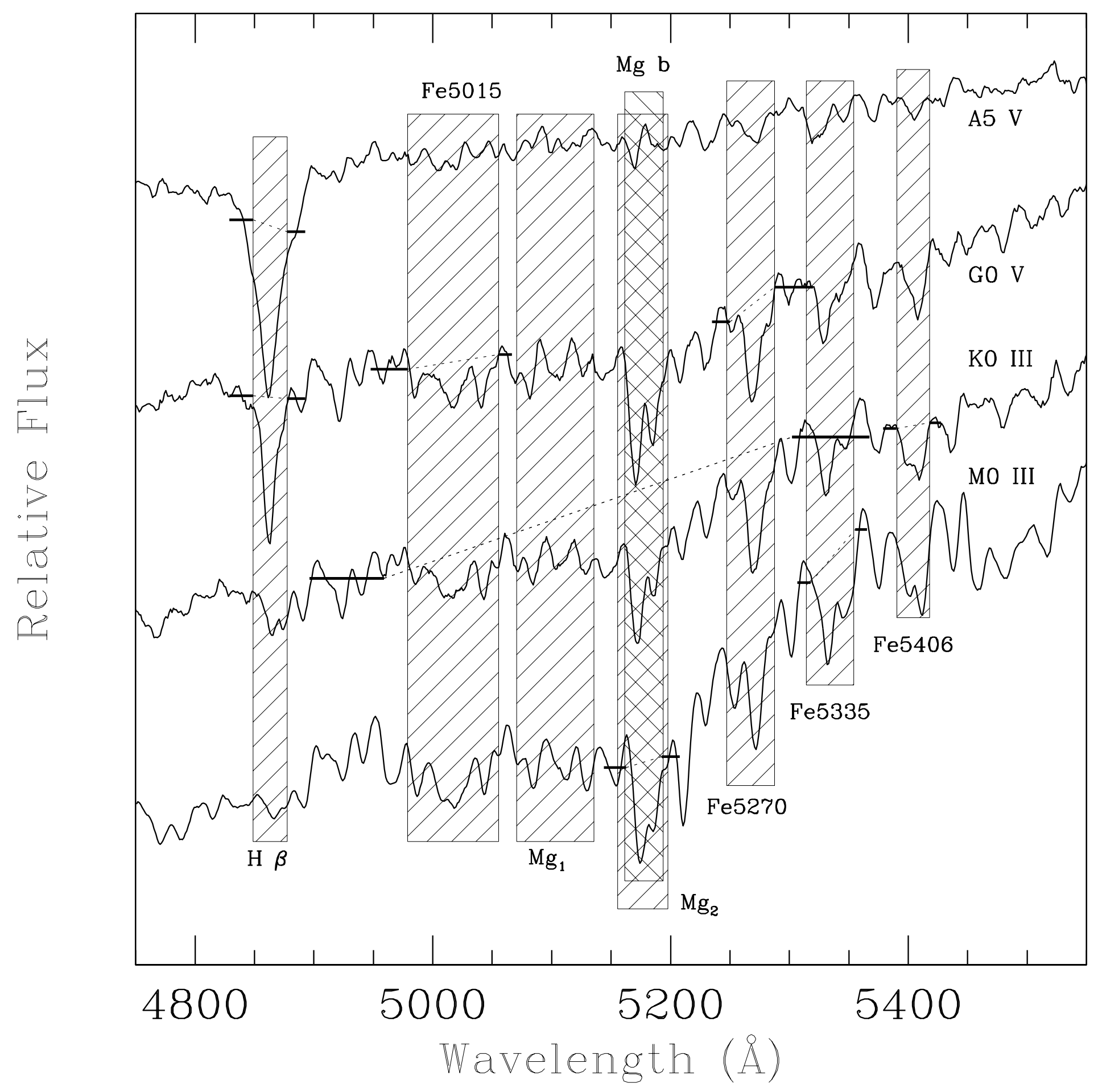




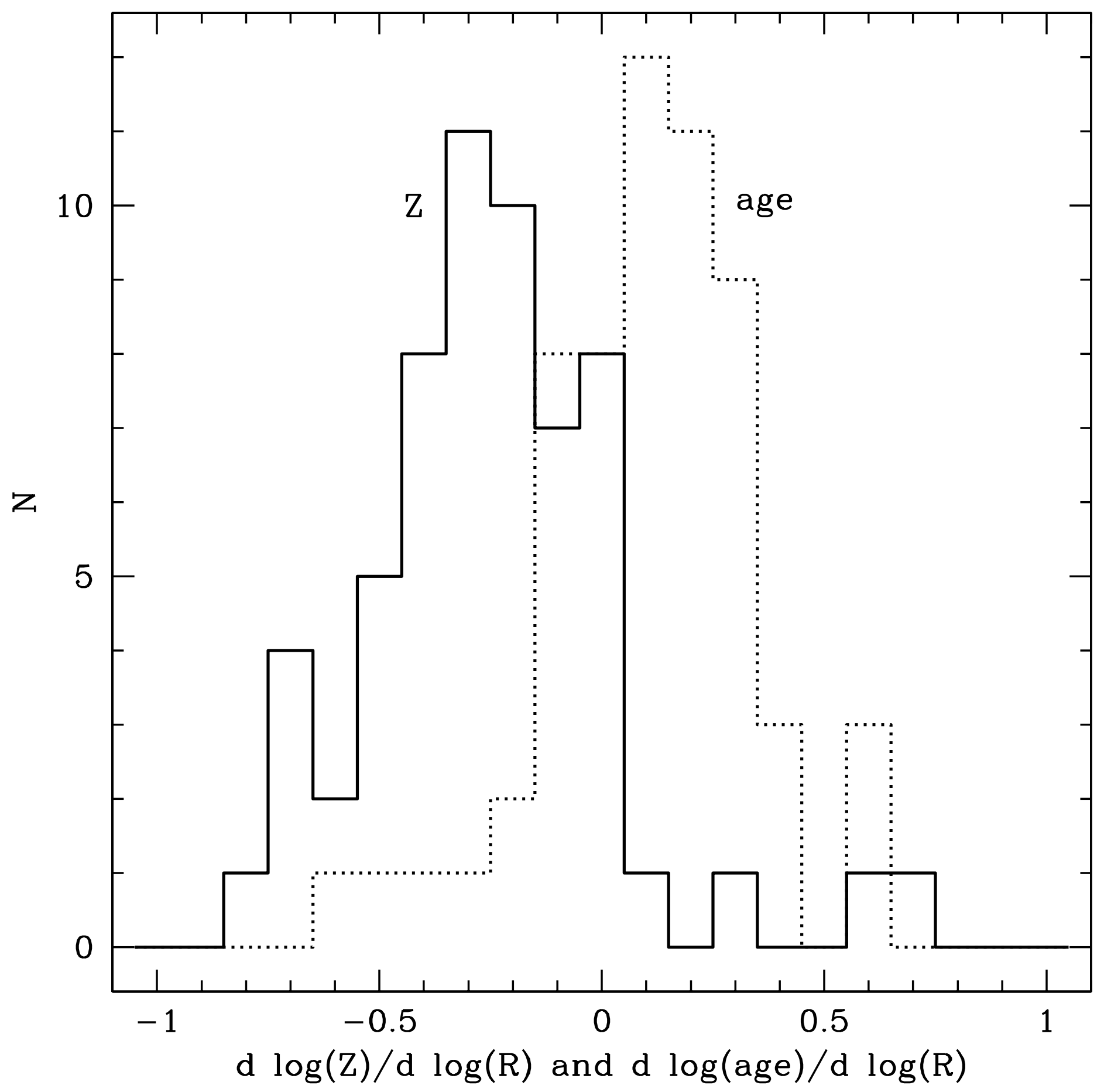




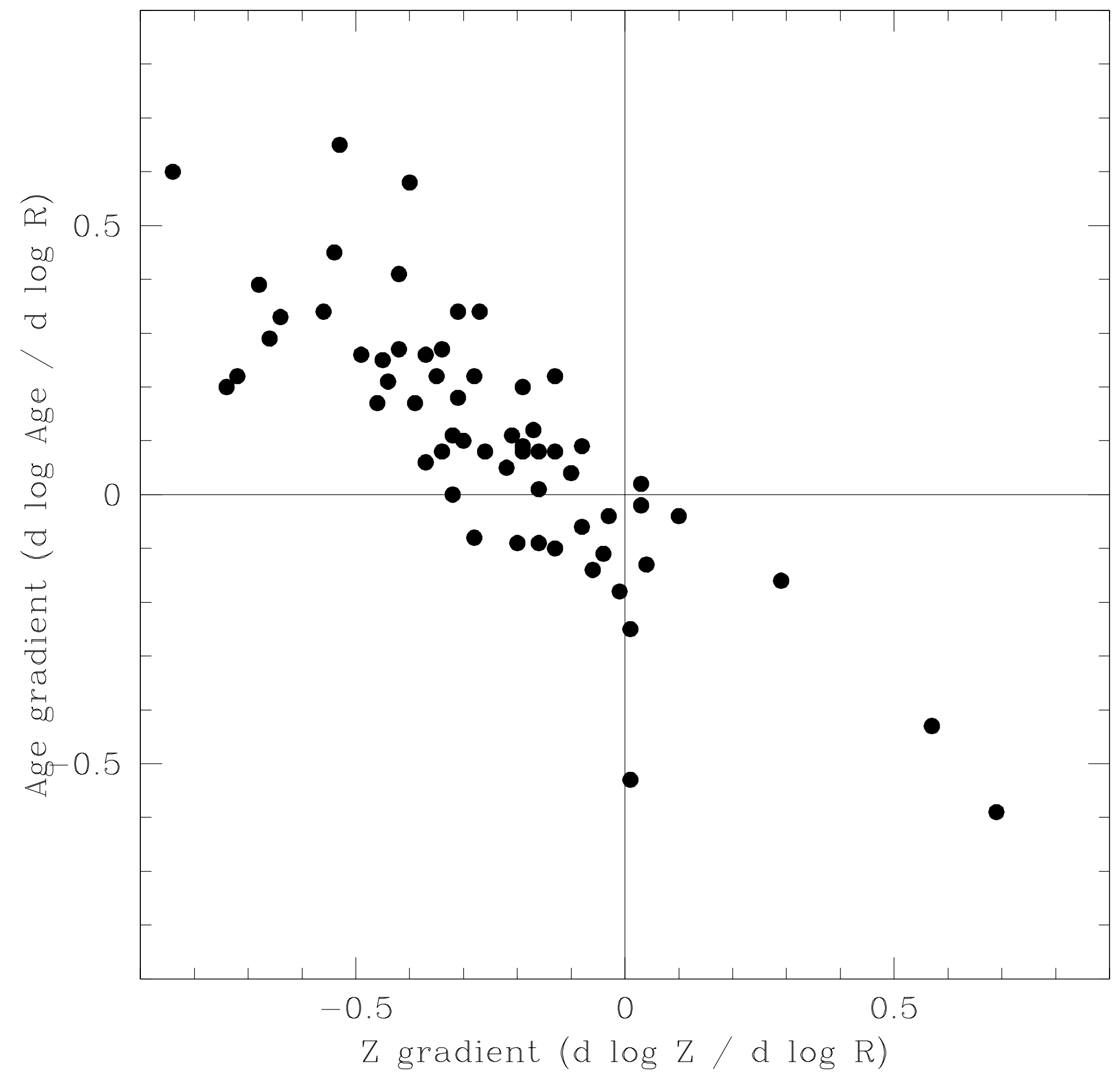




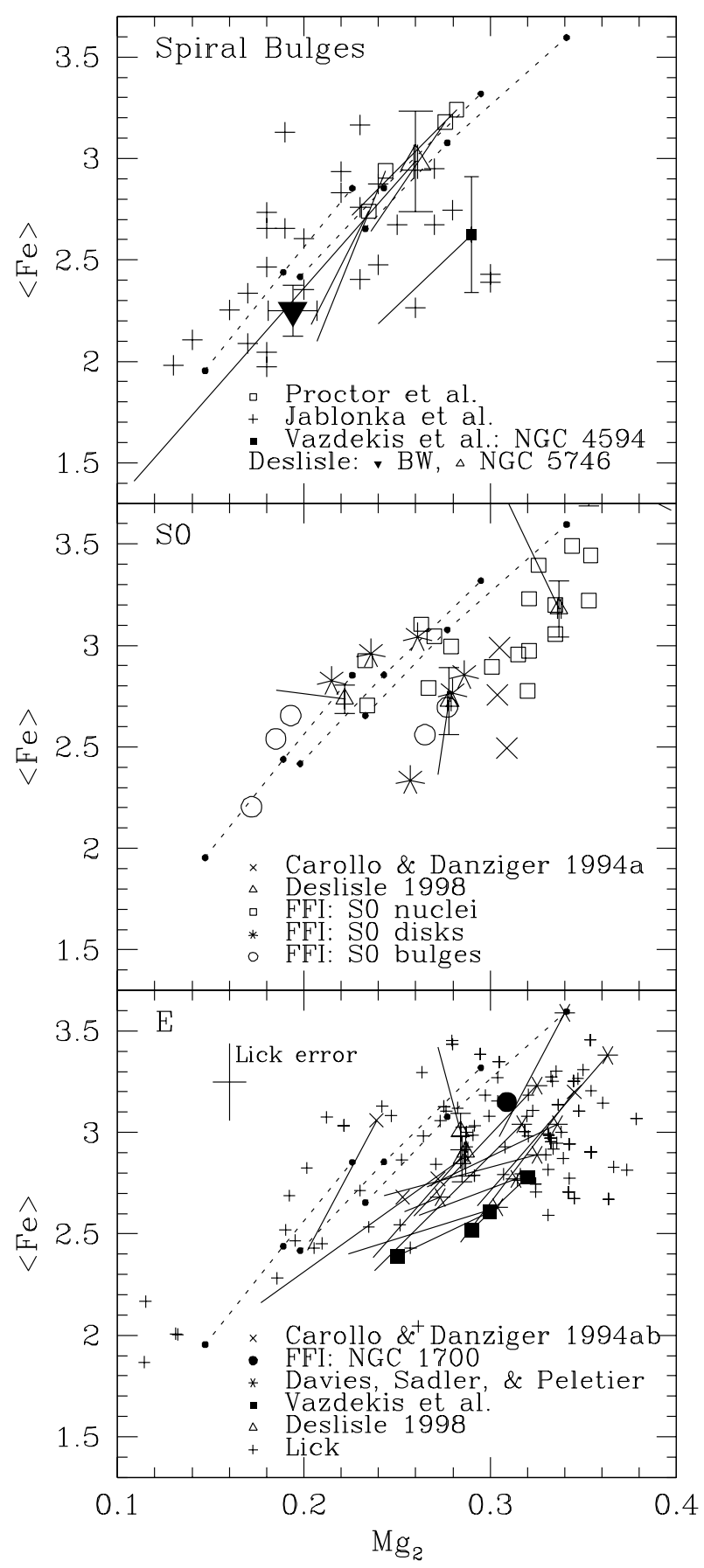




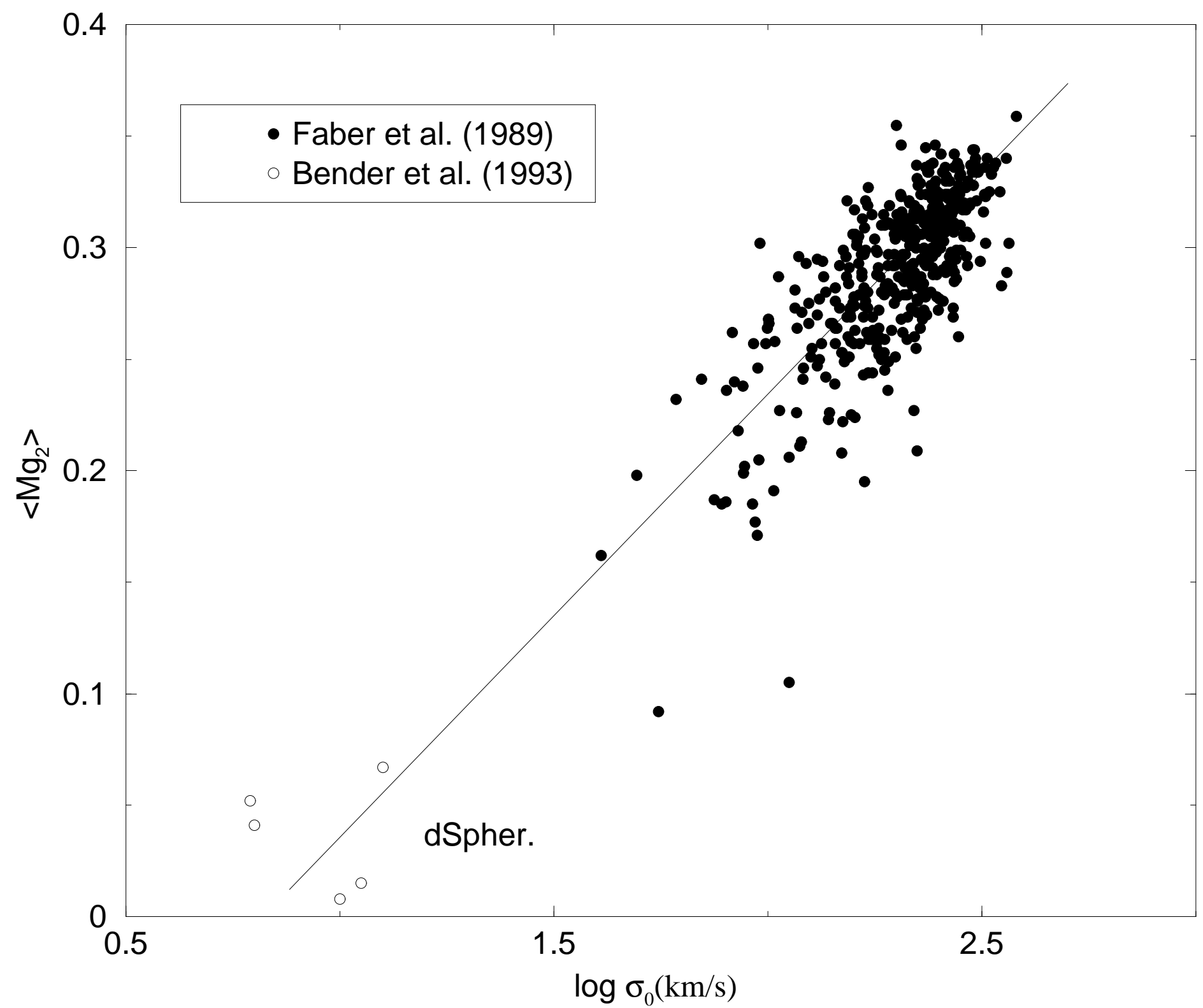



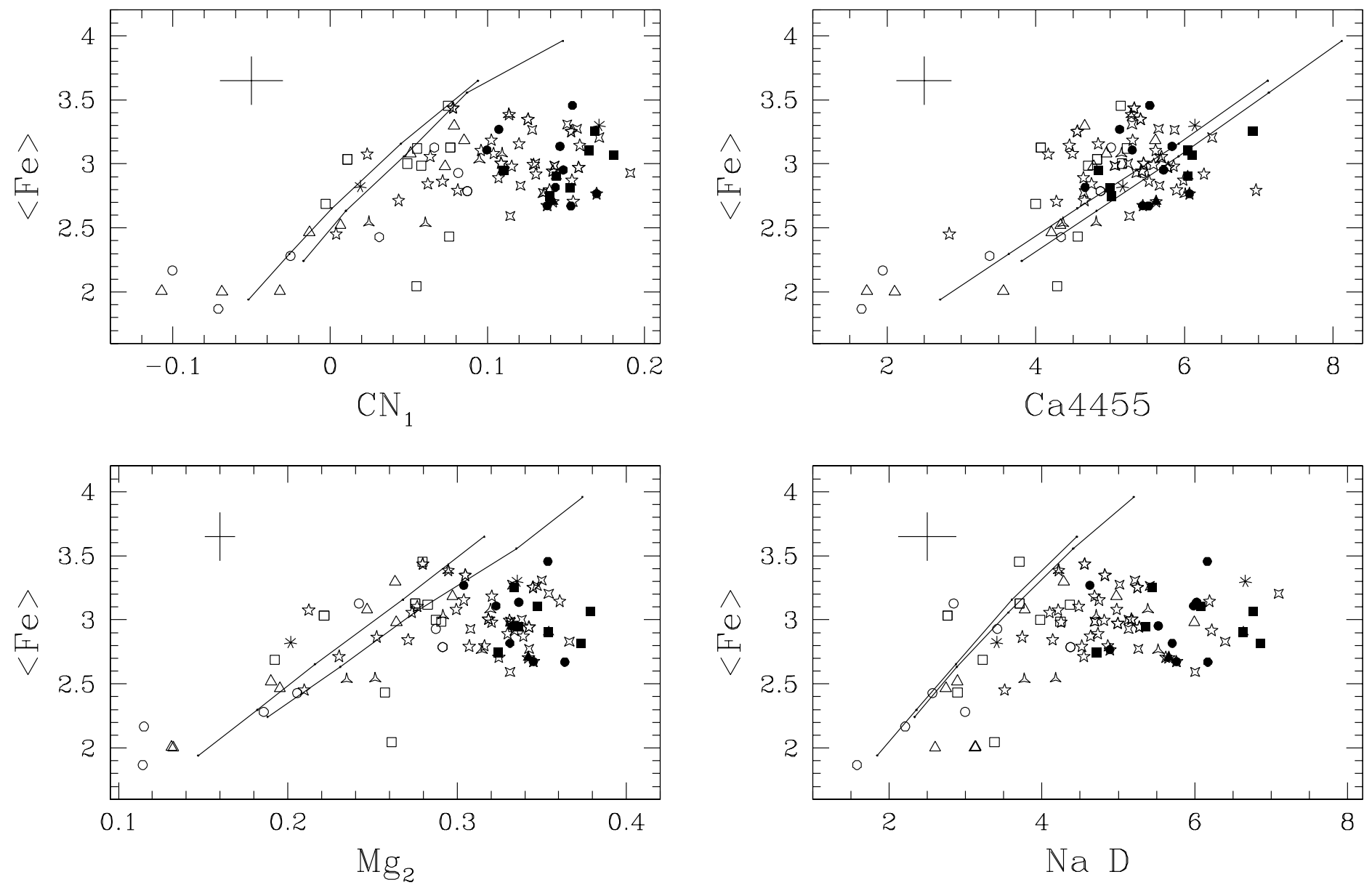